AREA IV - Undeformed sediments and late movement structures

Geographical setting: Weakly or non-deformed Cretaceous and Tertiary sediments cover the entire Danish sector outside the wrench zones (figs. 10, 13, 47).

Geological description: In Permian, Triassic and Jurassic times, the individual fault blocks of the Central Graben subsided differentially along normal faults. In Cretaceous and Tertiary times, however, this pattern changed into a more general non-fault controlled subsidence of the entire North Sea area. Therefore non- or weakly deformed Upper Cretaceous and Tertiary sediments cover large parts of the Danish North Sea. In these sediments, stratigraphic traps are expected to be present.

In some areas, however, late movements along old deep-seated faults resulted in uplift, which in some cases has created gentle structural arch traps. This mechanism is also termed 'drape' (Blair 1975).

Distribution of potential hydrocarbon traps: In nondeformed sediments, hydrocarbons may be trapped in primary stratigraphic traps. These are often expected to be located along structural highs, but e.g. Paleocene submarine fans cover most of the northern Viking Graben. Stratigraphic traps might be improved by differential compaction. This mechanism is based on the assumption that there is less lithostatic pressure on the crest of the reservoir body than on the flanks. As a result sediments on the flanks undergo greater compaction, and the vertical closure of the stratigaphical trap is improved.

Unlimited reservoirs and stratigraphic traps may in addition be weakly deformed by drape into gentle structural arch traps or late movement structures. Simple drape, as defined by Blair (1975), is caused by re-juvenation of deep-seated old faults, resulting in uplift and creating a gentle dome structure.

An example of this type of late movement structure is the Inge structure which is located over the crest of the Dogger High. This structure is characterized by a rather large areal closure and a relatively small vertical closure, which were probably induced by late movements along the Dogger High faults.

\subsection{Reservoir rocks}

\author{
By Finn Jacoben, Kirsten Lieberkind \& Erik \\ Nygaard
}

The present chapter contains a review of the potential reservoir rocks drilled in the Danish Central Graben. The source data for this descriptive summary originates primarily from wells drilled to explore chalk reservoirs. The main part of possible chalk reservoirs has been evaluated only by using wire line logs, since few cores have been cut and very few tests performed. Therefore, further investigations are necessary to evaluate and describe these possible reservoir rocks properly.

In this chapter, each zone in which shows have been recorded, and each porosity zone, is described. This is done with reference to the formations/log-units given earlier in the paper. The characteristics relevant to reservoir evaluation, such as the extension, thickness, depth, porosity, and permeability, are described. The characterizing terms (good, fair, etc.) used for reservoir parameters in sandstone reservoirs cannot be applied automatically to chalk reservoirs. The descriptions are accompanied by maps showing the known occurrences.

\subsection{CA-1 Unit (Early Carboniferous)}

Lithologic characteristics: Sandstone, siltstone, carbonaceous shales and sporadic coal seams.

Extension, thickness, and depth: According to Ziegler (1977), Carboniferous deposits are present in the major part of the Central Graben, but due to lack of data a delineation of the extension in the Danish sector is not possible. The thickness recorded in the P-1 well is $67 \mathrm{~m}$, but the regional thickness may well be 100 to 200 $\mathrm{m}$. Away from the highs, the deposits are mostly very deeply seated.

Reservoir parameters (fig. 49): Only the sandstones may be considered as potential reservoirs (net reservoir rock $13.5 \mathrm{~m}$ ). The sandstones vary from fine to medium-grained, occassionally silty. The porosity varies from 5 to $15 \%$ (average $11 \%$ ). Calcite cement has been found and may reduce the porosity.

Remarks: The section is stratigraphically below the 
known potential sources. Therefore the CA-1 Unit may represent a possible reservoir in faulted areas only.

\subsection{Rotliegendes Group}

Lithological characteristics: The lower section comprises volcanic tuff. The upper section consists of soft, and occasionally silty marl, interbedded with shale, and at the base sandstone.

Extension, thickness, and depth: The thickness of the Rotliegendes is, in the P-1 well, more than $200 \mathrm{~m}$, but may be more than $1000 \mathrm{~m}$ in the central part of the basin. Away from the highs the series is very deeply seated.

Reservoir parameters (fig. 49): Only the sandstones may be considered to have reservoir properties. They are coarse to fine grained and occassionally silty or shaly. Calcite cement is found in the lower beds. The total known thickness of the group is $213 \mathrm{~m}$, with a maximum net reservoir zone of $20.5 \mathrm{~m}$. The porosity is rather low (2-10\%, average $5 \%)$ due to cement and a varying content of clay and silt. The permeability is not known.

Remarks: No shows are found in the Rotliegendes in the Central Graben area, but gas finds are known from the southern North Sea, northern Holland, Germany, and Poland. The probable lack of source rocks below the Rotliegendes Group suggests that it may be a hydrocarbon-bearing reservoir rock in faulted areas, where it may be sourced from younger beds only.

\subsection{Zechstein Group}

Lithologic characteristics: This section is characterized by evaporites.

Extension, thickness, and depth: In the Central Graben, Zechstein deposits are mostly drilled on domal structures. Therefore the stratigraphy, lithology, thicknesses of the units, extension etc. cannot be clarified. In the basin between the structures the series is very deeply seated.

Reservoir parameters (fig. 50): Dolomitic intervals are the only possible primary reservoirs. From onshore wells the dolomite beds are known in some instances to have fairly high porosities and permeabilities. Often the pores in the dolomites are filled with anhydrite, which reduces the porosity to near zero.

Some cap rock anhydrites in Central Graben are fractured. Overlying oil may have migrated into these fractures, but permeability remains very low.

\subsection{Bacton Group (Early Triassic)}

Lithologic characteristics: Dominated by claystone which in some places can be silty, anhydritic, or calcareous. There are minor silt and sandstone beds.

Extension, thickness, and depth: The formation has been encountered in 3 wells only (B-1, Q-1, and U-1), but it is probably present in the entire area.

Seismic evaluation is possible only in the southern part, where the top Triassic varies in depth from 3000 to $4500 \mathrm{~m}$. To the north, the group grades into the Smith Bank Formation (Q-1).

The thickness is fairly constant and greatest near topographic highs. The thicker parts seem to consist of relatively coarse-grained sediments derived from the highs. The net sand thickness therefore seems to be a result of local geological processes.

Reservoir parameters (fig. 50): Gas shows are recorded in the Q-1 well, but the available data allow no statements regarding the reservoir conditions.

In the study area there is fair data only from the U-1 well. Here the porosities of the sand beds are 10 and $17 \%$, for the Bunter Sandstone and Bunter Shale respectively. The higher porosity in the intercalated Bunter Shale Formation sand beds may be due to a higher clay content, which would reduce the permeability. No data to clarify this question are available.

\subsection{J-2 Unit (Middle Jurassic)}

Lithologic characteristics: Interbedded pure sandstones, claystones and heterolithic sand-siltstones with coal seams.

Extension, thickness, and depth: The Unit may cover most of the Central Graben area, but it is until now known only in the A-2, M-8, O-1, and U-1 wells. Most of these wells are situated on domal structures. The influence of halokinesis on the thickness of the formation has not yet been clarified. In large parts of the 

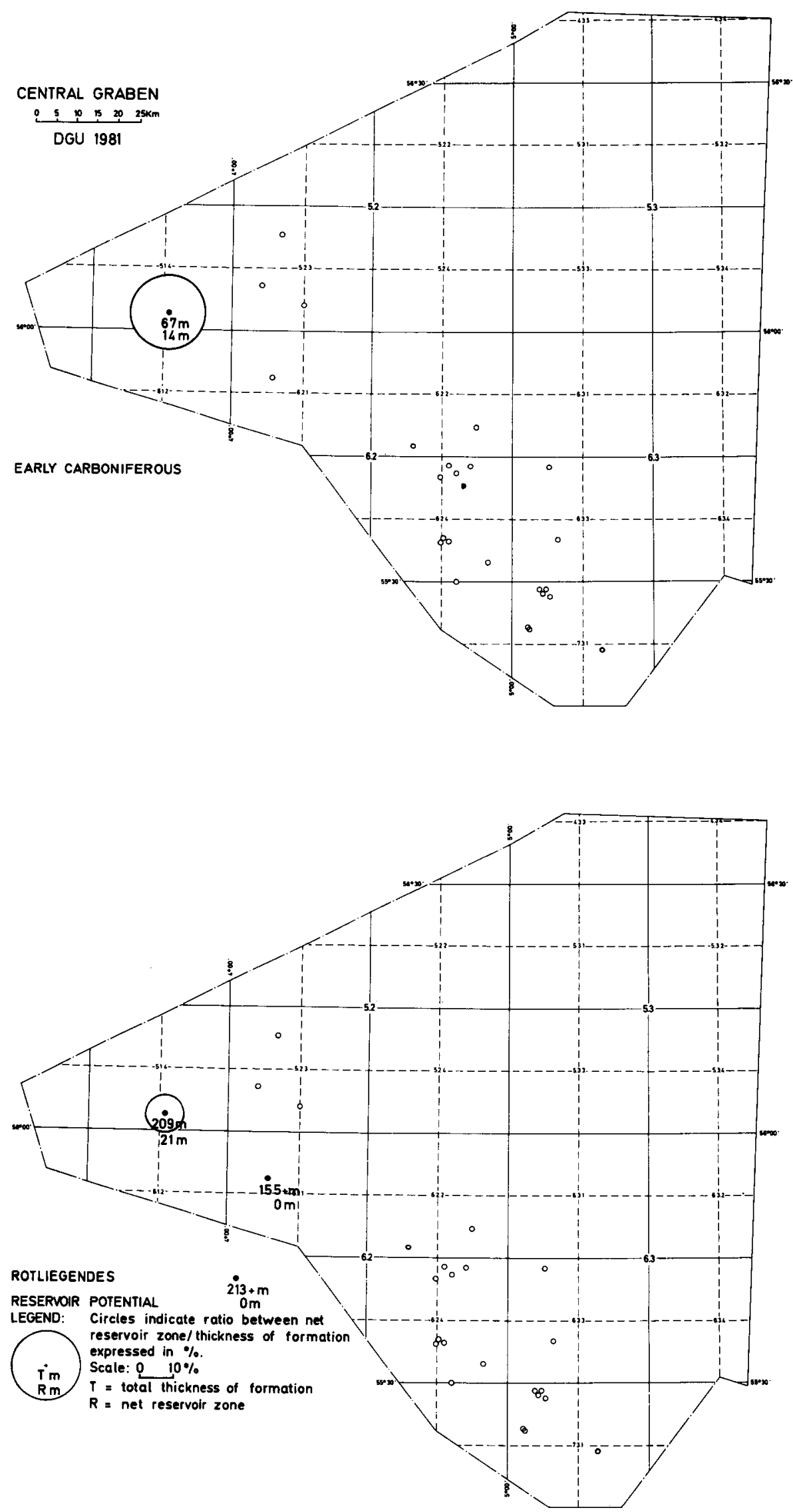

Fig. 49: The reservoir potential of a) Early Carboniferous and b) Rotliegendes section in the Central Graben area. 

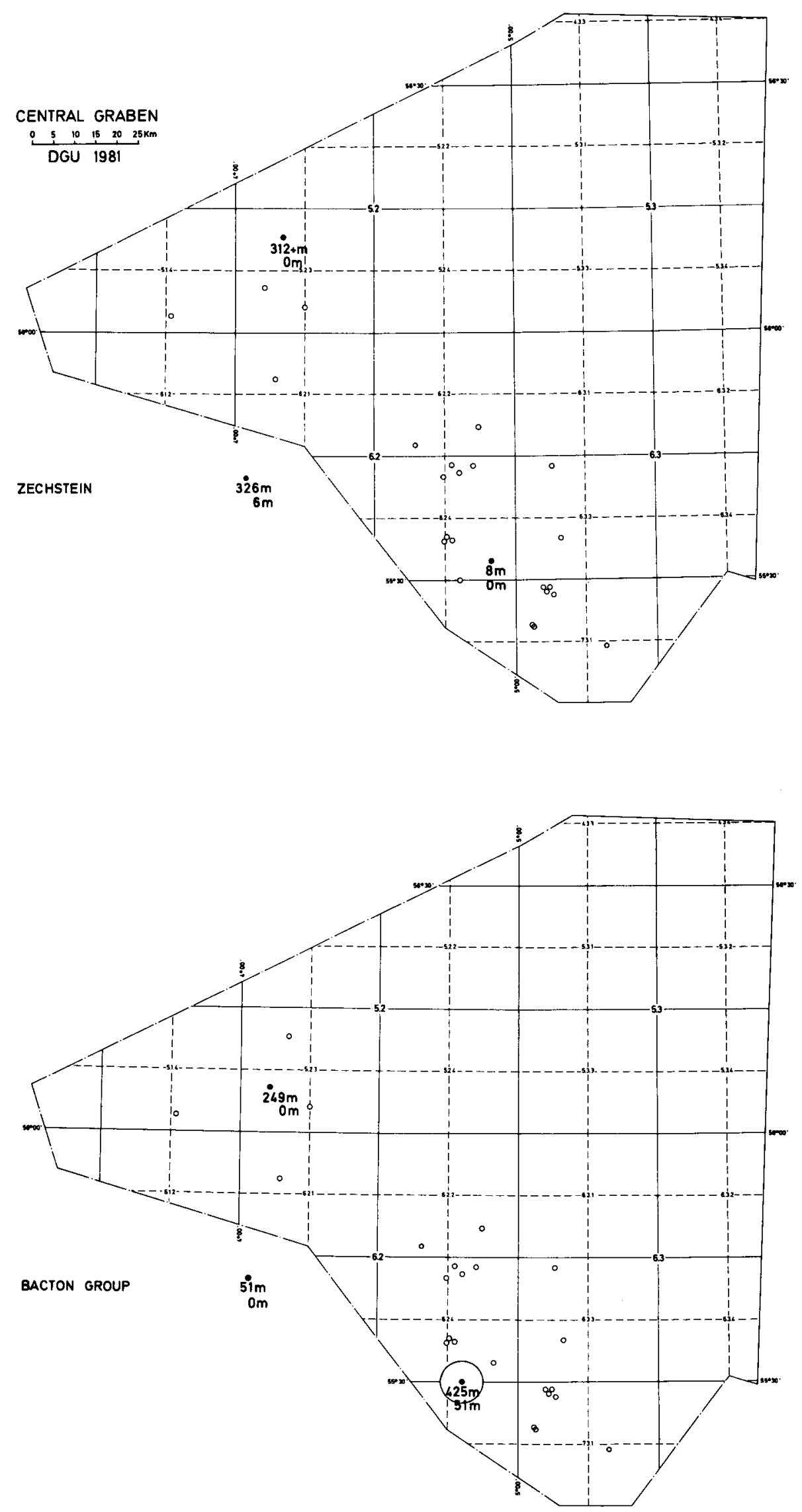

Fig. 50: The reservoir potential of a)Zechstein and b) the Bacton Group. - For legend, see fig. 49. 

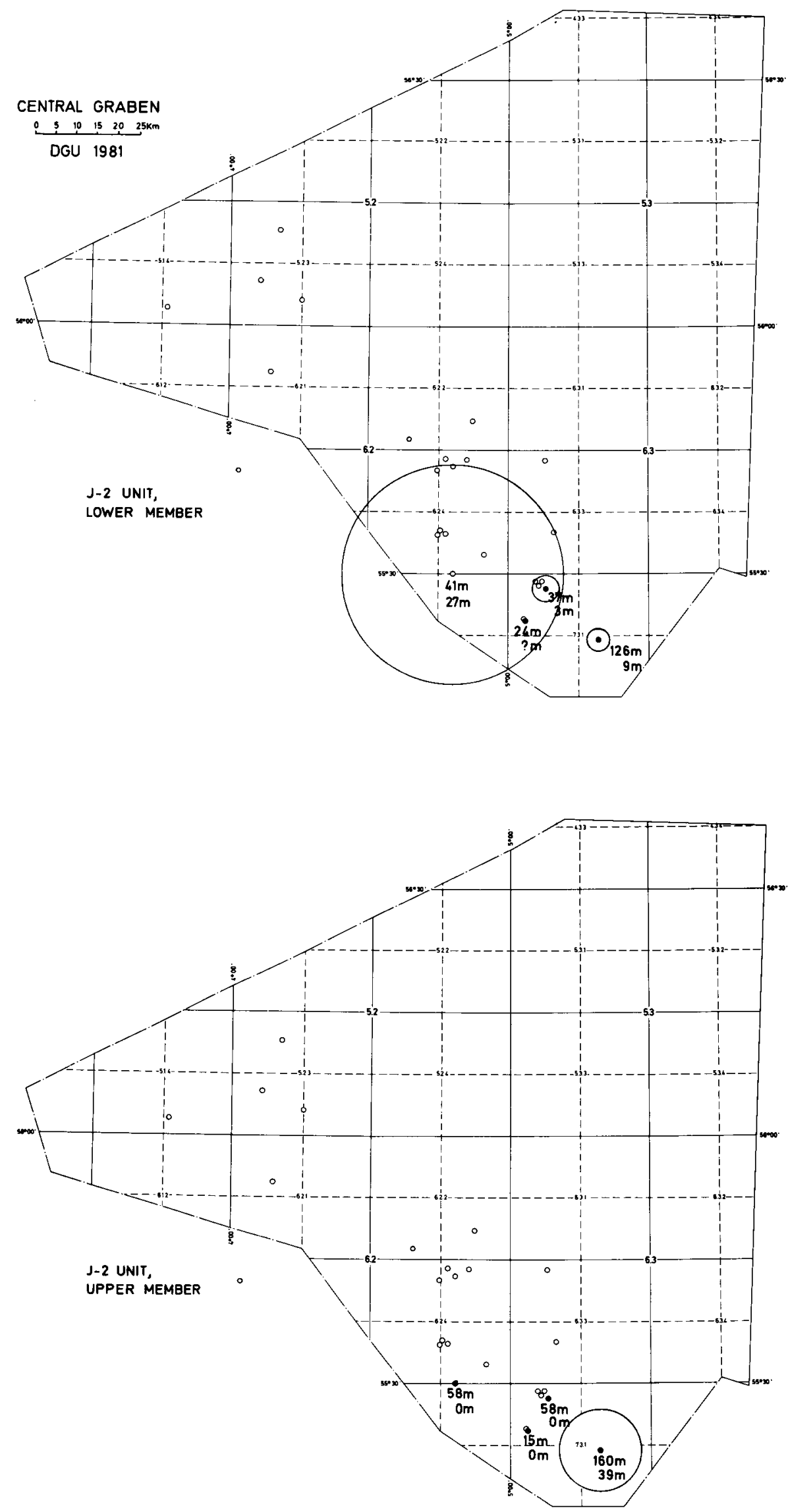

Fig. 51: The reservoir potential of a) the J-2 Unit, Lower Member and b) the J-2 Unit, Upper Member. For legend, see fig. 49. 

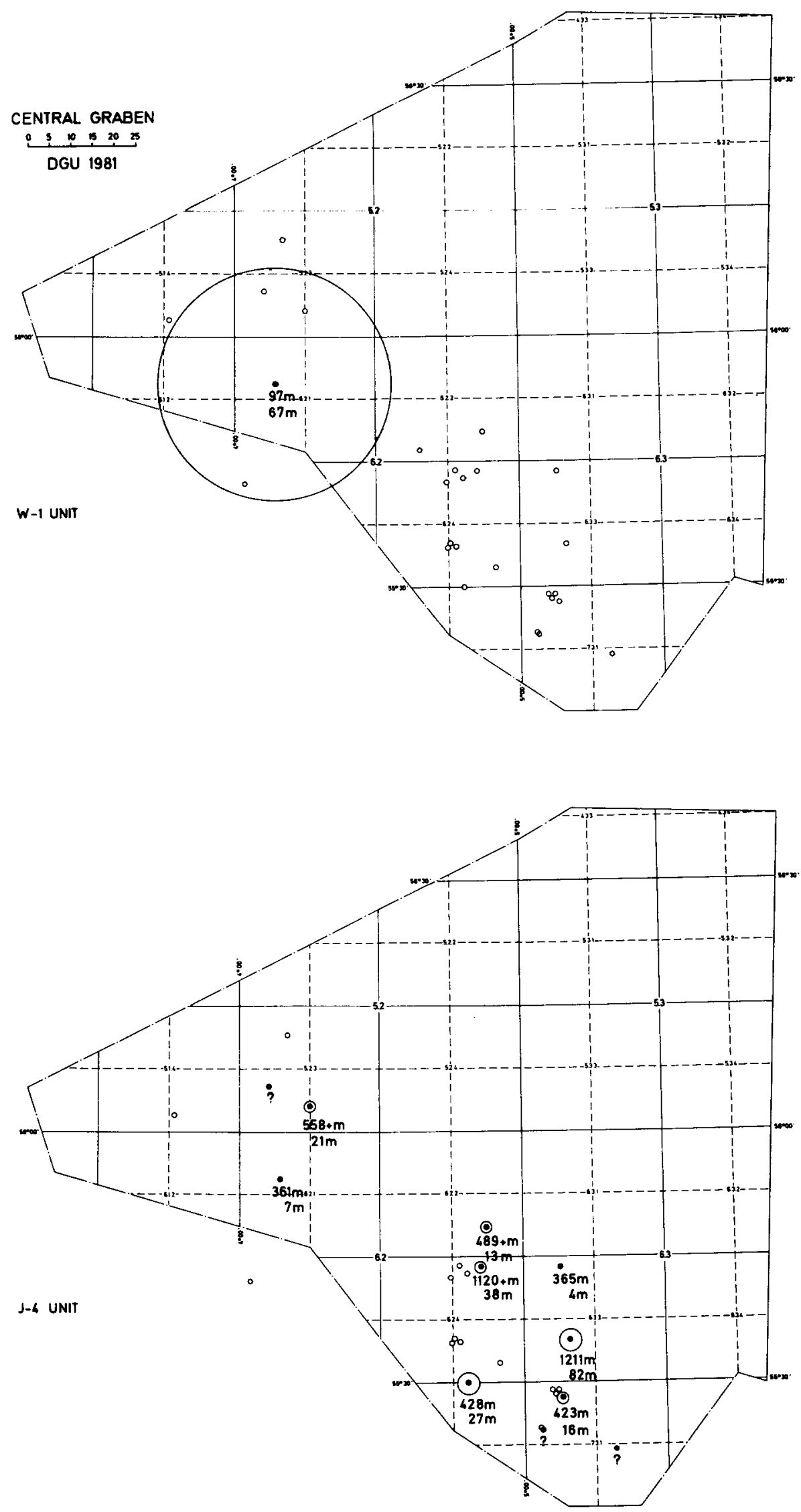

Fig. 52: The reservoir potential of a) W-1 Unit and b) the J-4 Unit. - For legend, see fig. 49. 

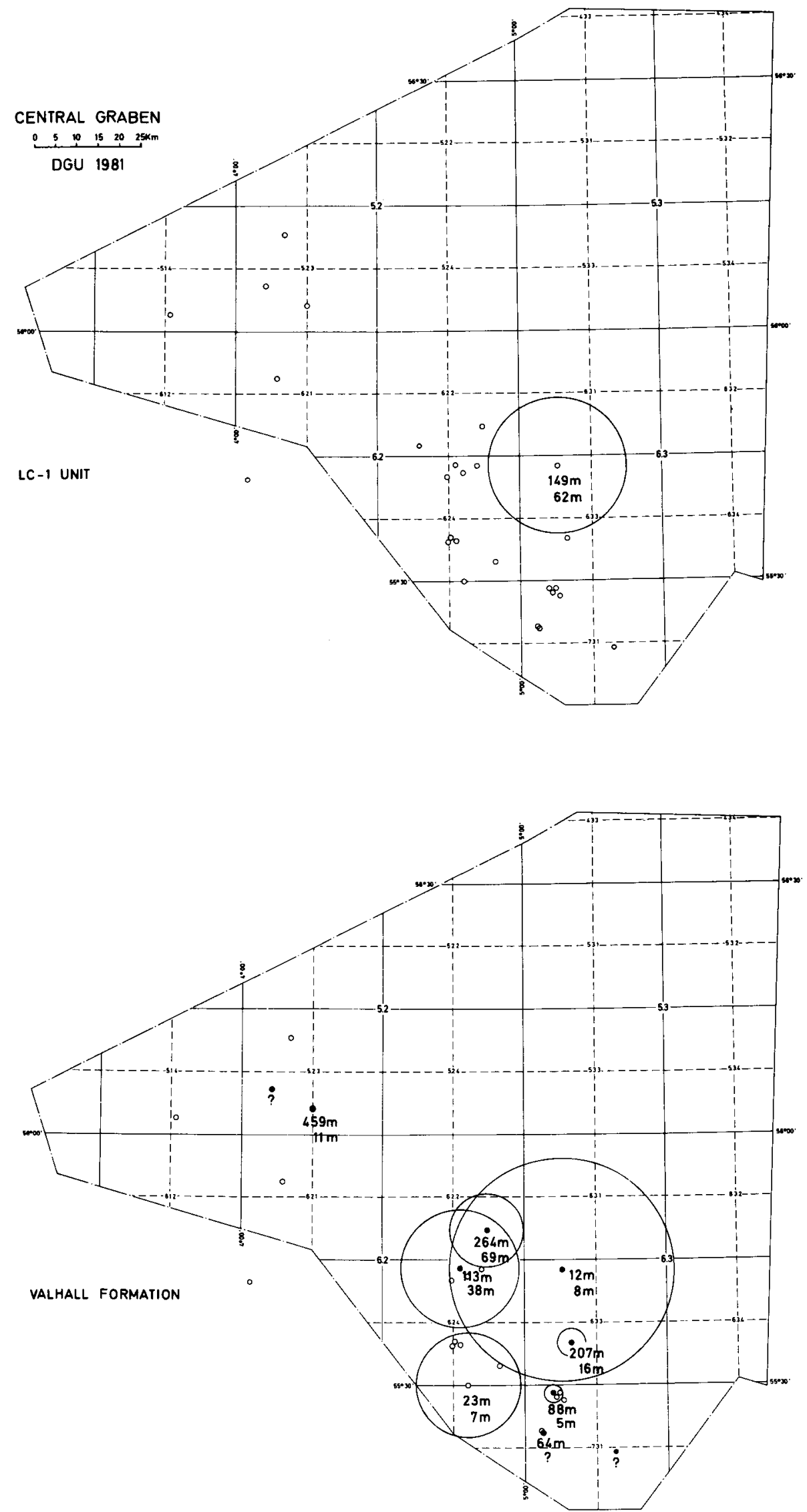

Fig. 53: The reservoir potential of a) the LC-1 Unit and b) the Valhall Formation. - For legend, see fig. 49. 
Central Graben (between the structures) the depth may be $4000-5000 \mathrm{~m}$.

Reservoir parameters (fig. 51): The Unit is divided into a lower member consisting of interbedded thick sandstones, claystones, and heteroliths and a less sandy upper member with coal seams.

In most wells there is a quantitatively upward decreasing occurrence of sandstone beds. This corresponds to the distribution of gas shows, which dominate in the lower member.

The following two facies may have reservoir properties: 1) the pure sandstone, which is hard to friable, fine to medium-grained, slightly calcareous with fair visible porosity, and 2) the heterolithic sand- and siltstones.

Only the sandstone beds are evaluated here. The average porosity is $20 \%$, varying from $15-30 \%$. Some sandy intervals are calcite cemented, which has reduced both porosity and permeability.

\subsection{W-1 Unit (Late Jurassic)}

Lithologic characteristics: The Unit consists of sandstones, minor siltstones, and conglomerates with interbedded, dark grey claystone and heterolithic silt- and claystones.

Reservoir parameters (fig. 52): All sections except the interbedded claystone seem to be potential reservoirs, with the conglomerates being the best reservoir rock. The conglomerates comprise rounded granular clasts of chalcedony and quartz, some dolomitic and granitic clasts and minor ammounts of siltstone clasts, all set in a medium-grained sand matrix.

The sandstones are fine-grained and firm, in part friable, depending on the degree of calcite-cementation. Loose medium-grained sand beds of vitreous and milky quartz have been found.

Three major sandstone intervals occur in the W-1 Unit, giving a net sand thickness of $67 \mathrm{~m}$ with a porosity varying from 6 to $14 \%$. The average value of porosity is $11 \%$ based on bulk density log. The neutron porosity seems to be higher due to the clay content.

Two Formation Interval Tests (FIT) were performed in the W-1 Unit but no conclusive calculations of the permeability can be made. The moderate sorting and the presence of clay in the sandstone will lower the permeability.

\subsection{J-3 Unit (Late Jurassic)}

Lithologic characteristics: Claystone, often slightly silty, slightly calcareous; and subordinate occurrences of siltstone, medium to coarse-grained; and marlstone.

Reservoir parameters: Oil traces are reported from silty and calcareous layers in the A-2 and G-1 wells. Therefore silty layers may have reservoir properties, whereas the dominating clayey intervals have none. The irregular occurrence of the silty and calcareous beds may indicate restricted possibilities for commercial finds. These beds may be found along domal structures, on the top of which the J-3 Unit is absent or exists only as rims around the highs.

No cores have been cut and no tests performed in this Unit.

\subsection{J-4 Unit (Late Jurassic)}

Lithologic characteristics: Claystone, shaly, laminated, slightly silty and calcareous, with mica. Interbeds of thin lime- and dolostones are numerous.

Reservoir parameters (fig. 52): Several hydrocarbon shows have been reported from the lime- and dolostone stringers and the more silty intervals. The stringers may be of diagenetic origin and contain secondary porosity, contributing to the creation of reservoir conditions. The thickness of the stringers is approximately 2'. The log porosity in the stringers varies from $3-36 \%$, averaging $10-12 \%$. The total number of stringers varies from well to well.

The Unit is tested only in one well (E-1). The test indicated no significant amount of oil or gas. The permeability cannot be evaluated due to poor quality of test data.

The porous stringers may be sourced by the Unit itself.

\subsection{LC-1 Unit (Late Jurassic - Early Cretaceous)}

Lithologic characteristics: Sand and siltstone, silty sandstone, and sandy siltstones, with thin subordinate beds of calcareous shale and clay.

Extension, thickness, and depth: The Unit has been found in the V-1 well only, which is situated close to the margin of the Ringkøbing-Fyn High. Here the 
thickness is $61.5 \mathrm{~m}$ and depth $2702-2851 \mathrm{~m}$. The Unit may be found as a fringe around other, similar structures.

Reservoir potential (fig. 53): The entire Unit seems to be a potential reservoir rock. This is confirmed by traces of oil and gas in the uppermost silty and sandy interval.

The porosity varies between 3 and $36 \%$ (log) with an average of $22 \%$. No cores have been cut and no tests have been performed in the Unit.

\subsection{Valhall Formation (Early Cretaceous)}

Lithologic characteristics: Soft, grey to dark grey calcareous mudstone and shale with abundant limestone, marl, and siltstone beds.

In the lower part, the Formation has a relatively high terrigeneous content and may in parts be submarine fans. Dolomitic stringers (diagenetic) similar to those of the J-4 Unit are found in this lower part. The upper part of the Formation consists of lime and marlstone.

Extension, thickness, and depth: The Formation is the most prominent part of the Lower Cretaceous in the Danish Central Graben. The thickness of the Formation appears to show local maxima to more than 600 $\mathrm{m}$, depending on the setting in relation to halokinetic structures. The Formation is generally thin or absent on structural highs.

Reservoir parameters (fig. 53): Due to the location of the Danish wells on salt domes, very little data are available on deep seated parts in the study area. However, in the V-1 well the sand- and siltstones interbedded with claystones might indicate good reservoir conditions. The porosity varies from 19 to $24 \%$ ( $\log$ ), averaging $22 \%$.

The lime- and marlstones in the upper part of the formation may also have good reservoir properties, and shows are found in more wells.

The few tests from this Formation (the Adda-1 and E-1 wells) indicate relatively poor reservoir conditions.

\subsection{Rødby Formation (Early Cretaceous)}

Lithologic characteristics: The lithology varies consid- erably from well to well, but the main characteristic features of the Formation are marls, limestones or calcareous mudstones interbedded with clays and shales. Sandy intervals also occur.

Extension, thickness, and depth: The Formation is widely distributed in the Central Graben outside structural highs. The Formation is generally thin, typically 10-20 m, although local thicknesses up to 50 $\mathrm{m}$ may occur (the E-1 and I-1 wells). This greater thickness may be a result of sand deposition as indicated in the E-1 well.

Reservoir parameters: Apparently this Formation has limited reservoir potential. However, shows in sandy intervals in E-1 may indicate a small reservoir potential.

The porosity varies from $1 \%$ to $40 \%(\log )$.

A drill stem test in the E-1 well gave no flow, indicating a very low permeability.

\subsection{Chalk Group (Late Cretaceous - Early Cenozoic)}

Exploration for oil and gas in chalk reservoirs, has been accelerated within the last ten years by discoveries of successful hydrocarbon reservoirs in the chalk in both the USA and the North Sea. Although chalk is a fairly poor reservoir rock, high production rates can be achieved if fracturing and a high oil column are present. The production can be enlarged by mechanical fracturing and/or acidizing of the rock, and the Chalk Group in general can be considered a reservoir rock (Scholle 1977b). More than $90 \%$ of the chalk is normally composed of calcareous nannoplankton (coccoliths) and foraminifera. Other biogenic- or terrestrial constituents such as bryozoans, clay-minerals, and clastic sand-grains are present in minor amounts.

The sediment is very fine-grained, as the sizes of the coccolith platelets range from 5-30 microns, and disintegrated platelets are even smaller. The foraminifera are found in the silt and sand fractions. The chalk provides a good primary porosity, but the narrow pore throats result in a low matrix permeability.

The coccolith- and foraminiferal skeletal calciumcarbonate has a low magnesium content, which makes the chalk somewhat resistant to the diagenetic alterations that cause loss or gain of porosity. Nevertheless, several factors affect the porosity.

Compaction and diagenesis: Chalk, upon initial depo- 
sition, has approximately $70 \%$ porosity. The initial porosity decreases with depth of burial resulting in a porosity value of $5 \%$ at a depth of $2500 \mathrm{~m}$, following a 'normal compaction' trend (fig. 54). Several other factors affect the magnitude of porosity loss during the burial: Primary composition, tectonic stress, pore fluid pressure, the presence of hydrocarbons, pore water chemistry, and redeposition.

Primary composition: Variations in grain size, faunal composition, and clay content, result in differentiated diagenetic alterations, which cause variations in porosity and permeability.

The lower member of the Chalk- 6 Unit, often has a clay content of $10-15 \%$, which seems to prevent stylolitization and micro-fracturing. The clay occupies part of the pore space and blocks the pore throats.
This causes a low porosity and permeability. The primary content of silicious skeletons, which during diagenesis is dissolved and reprecipitated as chert, also reduces porosity and permeability. In some places chert layers may even be permeability barriers, as seen at the Dan field.

A high content of calcispheres, as often found in the Chalk-4 Unit seems to cause a slight reduction in porosity and permeability.

The coccolith-foraminiferal chalk of the Chalk-5 Unit and the upper member of the Chalk- 6 Unit, which seem to be the diagenetically most stable, has resisted porosity reduction at shallower depth. At greater depths of burial these units show abundant pressure solution (stylolites) as well as some reduction in porosity and permeability.

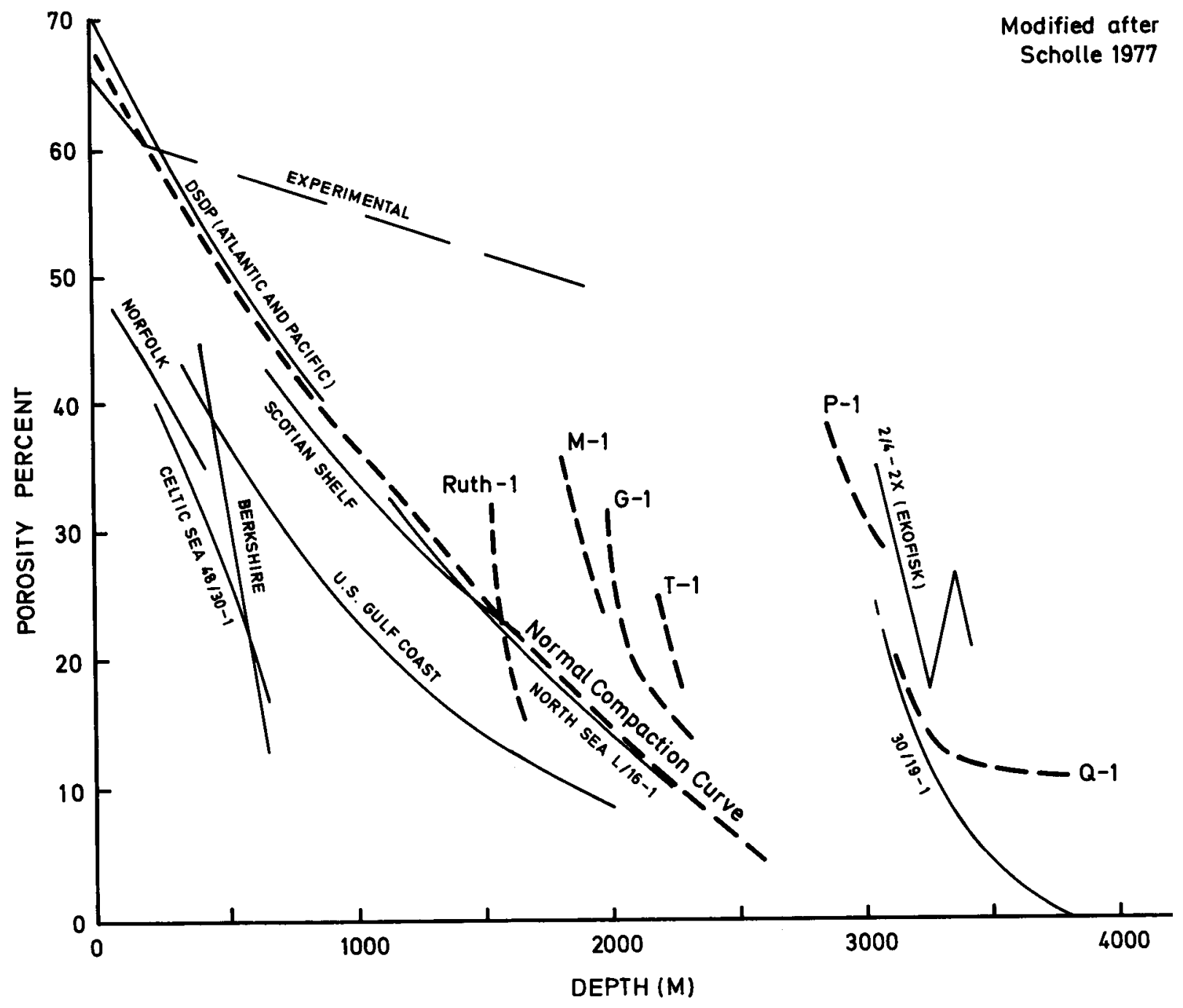

Fig. 54: Porosity v.s. depth of the Chalk Group in the G-1, M-1, P-1, Q-1, and Ruth-1 wells and a normal compaction curve plotted for comparison on a diagram from Scholle (1977b). 
Tectonic stress: In heavily faulted or folded areas, stresses may be introduced causing reduction in porosity and permeability, similar to that of overburden. The tectonic stress may, however, also cause fracturing. Although the porosity increases only by a few per cent by fracturing, the permeability increases by a factor of 100 or even 1000 . This is exemplified in the Ruth-1 well, drilled on top of a salt structure.

Pore fluid pressure: Mechanical and chemical compaction is dependent on expulsion of pore fluids. If the expulsion is obstructed, the pore fluid pressure will become higher than the hydrostatic pressure, grain to grain contact will be reduced and pressure solution will cease. In this way primary porosity and permeability can be preserved at much greater depths than those expected from 'normal compaction' curves. This is exemplified in the Dan field, where the fluid pressure is about 500 psi higher than the normal hydrostatic pressure, and the porosity is two times higher than that expected from the depth of burial (fig. 54). In the Danish Central Graben area, the Chalk Group is generally overpressured. The overpressuring is met at a depth of approximately $1200 \mathrm{~m}$, close to top Middle Miocene. The overpressured interval seems to include the Chalk Group and part of the underlying shales.

Presence of hydrocarbons: Early migration of hydrocarbons into the chalk reservoirs reduces loss of porosity during further burial, by decreasing the water content and thereby slowing down its reaction with the rock. This effect cannot be fully evaluated for the Chalk Group in the Central Graben area, as it might be masked by the effects of pore fluid pressure. Oiland water-wet chalk reservoirs are very similar in their porosity-depth distribution.

Pore water chemistry: The influx of fresh water into chalk tends to accelerate the loss of porosity and permeability. However, this has not been encountered so far in the Danish Central Graben area. The formation water hitherto found has either a normal marine salinity, or a higher chloride concentration. Moreover, salt-saturated brines have been found in wells situated on top of salt piercement structures. Alteration of chalk to dolomite, and associated porosity loss or gain, has been observed only in a few cases.

Redeposition: Allochtonous deposits may vary considerably in thickness and may be difficult to recognize in uncored intervals. In redeposited intervals higher porosities are often encountered, as compared to in situ chalk (R.F.P. Hardmann, pers. comm. 1981). Until now, slumped intervals have been recognized in only few wells in the Danish Central Graben; they may, however, be more common. Provided that the possible distribution areas of the slumped sediments and their nature are better known, there may be a chance of finding prospective reservoirs in allochtonous chalk.

Porosity distribution: In general porosity decreases with depth of burial (fig. 55). The average trend shows a decrease from $40 \%$ porosity at $1800 \mathrm{~m}$, to $5 \%$ porosity at $3650 \mathrm{~m}$. However, the total range for all wells is from $46 \%$ to $3 \%$ porosity.

The porosity versus depth distribution has a hourglass shape (fig. 56). The depth interval from 1768$2072 \mathrm{~m}$ is a high porosity zone with a porosity range from 14-46\%. Another high-porosity interval is found from $2713-3109 \mathrm{~m}$, where the porosity ranges from 2 to $38 \%$. In between the two high porosity intervals, from 2347-2499 $\mathrm{m}$, a rather narrow scatter of porosity values from $18-26 \%$ is encountered. From 3109 m, where the porosity range is $10-19 \%$, the porosity gradually decreases downwards to much lower values. This indicates that chalk buried deeper than $3100 \mathrm{~m}$ is probably a poor reservoir rock. Chalk at depths shallower than $3100 \mathrm{~m}$ is considered a fairly good to good reservoir rock, since it has preserved much of its original primary porosity. In contrast to the small general decrease of porosity downwards, there is a considerable porosity reduction downwards in all individual wells (fig. 55).

Average porosities of the Chalk Units generally decrease with depth of burial. This reduction, however, is relatively small in the upper member of the Chalk- 6 and Chalk- 5 Units. Therefore these units are considered to be the best resevoirs within the Chalk Group from a matrix porosity point of view.

In fig. 57 the porosity ranges within the deposit zones are plotted against depth of burial. Going from deposit zones 2 to 3 , both the structural elevation and the porosity increase. This can be explained as a depthdependant porosity distribution. In deposit zone 4 , the Chalk Group spans depths from above as well as below the values encountered in deposit zones 2 and 3 . The porosity range in deposit zone 4 , however, is smaller. As most of the reservoirs in deposit zone 4 are situated upon salt piercement structures, tectonic stress may have caused the reduction of up to $15 \%$ in porosity in this chalk, which is located equally or even structurally higher than deposit zone 3 .

Deposit zone 5 encounters the deepest situated chalk reservoirs and the lowest porosities. The upper 


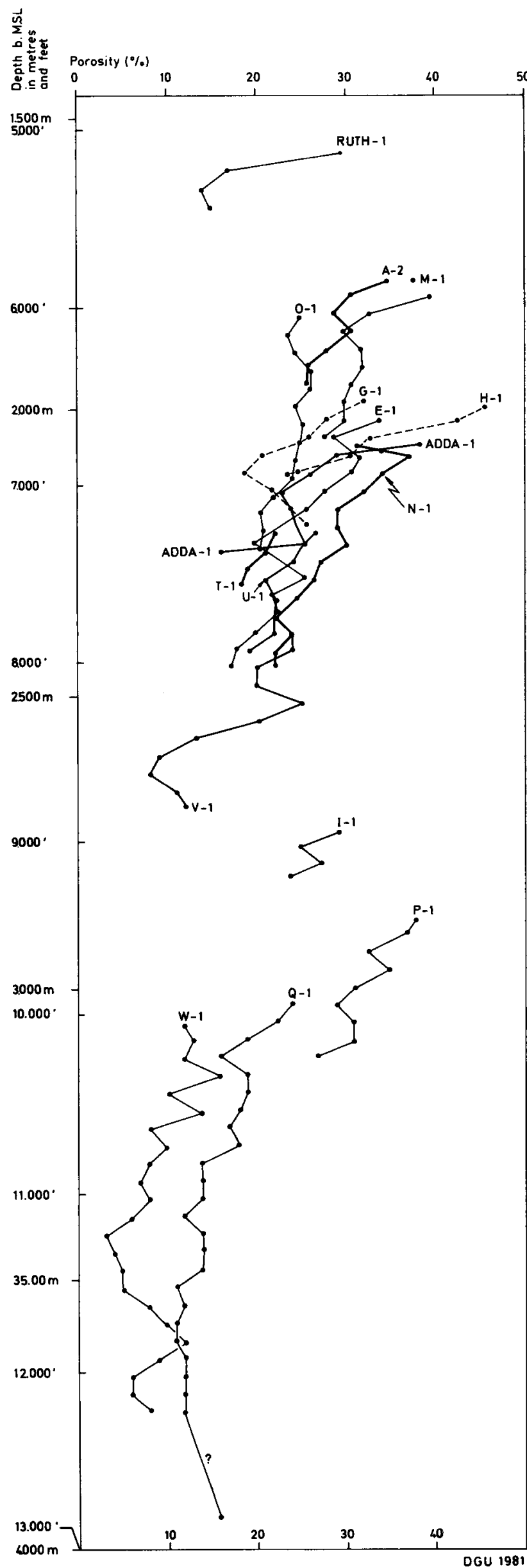

Fig. 55: Porosity v.s. depth plot of the Chalk Group in selected Central Graben wells.
$103 \stackrel{\overrightarrow{\mathrm{n}}}{\sum_{0}^{\mathrm{n}}}$

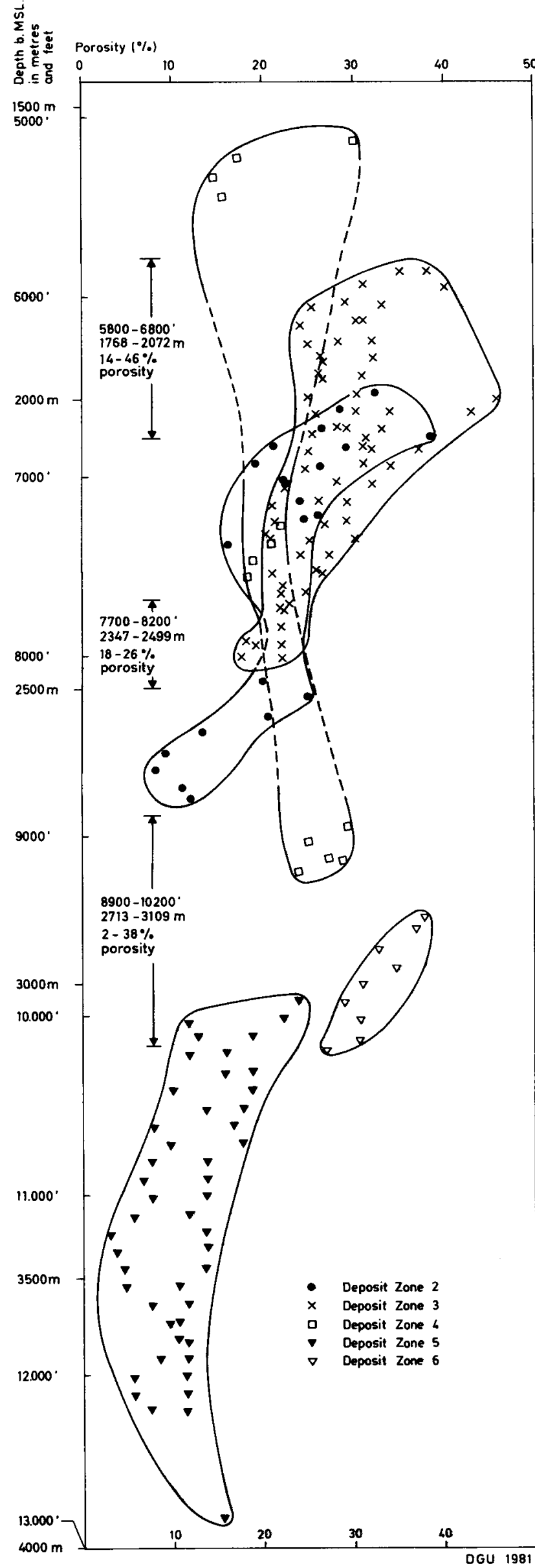

Fig. 56: Porosity v.s. depth plot of the Chalk Group. Data points are grouped and related to the Chalk deposit zones in Central Graben (see fig. 26). 
parts of these reservoirs, however, still have fair porosities, similar to the lower values of deposit zones 2,3 , and 4.

Chalk in deposit zone 6 is found in a narrow depth interval. These reservoirs are deeper seated than in deposit zones 2, 3, and 4, but equal to the upper part of deposit zone 5. Despite the deep situation the porosities are as high as the higher values of deposit zones 2,3 , and 4 , and they may be explained by formation water overpressure.

Permeability: As explained above, the primary permeabilities of chalks are low. Measured primary core permeabilities (air flow) generally range from 0.01 to $20 \mathrm{mD}$.

The permeability values vary greatly and show no clear relation to depth within the Chalk Units, (fig. 58). It is seen that the permeabilities in the two members of the Chalk- 6 Unit cover the same range. Permeability values from the Chalk-3 and 4 Units have a very narrow range despite depth, but still within the range of the Chalk-6 Unit. This distribution, however, is based on much sparser data than those of the overlying units. The Chalk-5 Unit has the highest permeabilities encountered, and they fall allmost entirely outside the field of the other units.

Judged from the matrix permeability distributions described above, the Chalk-5 Unit holds the best reservoir conditions within the Chalk Group.

Porosity versus permeability (fig. 59): Matrix permeability sometimes correlates with matrix porosity as a function of depth. This is seen as a straight-line relationship on a semilog plot. In several cases, however, this relationship cannot be confirmed. This other relationship is probably caused by microfracturing of the chalk.

The existence of fractures has been indicated through comparisons between core permeabilities and permeabilities calculated from tests.

Until now, the presence of fractures has been confirmed by production tests performed in the following wells:

Strongly fractured: Ruth-1

Moderately fractured: T-1, N-1, Adda-1, H-1, I-1

Not fractured: G-1, E-1

The remaining wells in the Danish Central Graben area either have not been tested or permeabilities have not yet been calculated.
Fracture distribution: The fracture distribution seems to be correlatable with the degree of structural elevation. Strong fracturing is found on the high Ruth saltpiercement structure. Moderately fractured chalk reservoirs are generally found on low salt piercementor shale flow structures, which are mainly found in deposit zones 2, 3, and the northern part of 4 .

Exceptions from this general tendency, are the T-1 and Adda-1 wells. The T-1 well is situated on a salt piercement structure, but the fracture permeability is found to be very low. It is possible that this situation simply reflects the fact that only the lowermost part of the chalk section, where open fractures are less likely to exist than in the upper part, has yet been tested.

Tests suggest that the chalk section in the Adda-1 well is fractured. Unfortunately, no core-data are available for comparison. Adda-1 is drilled on a flattop chalk closure, below which base chalk has a pronounced structural elevation. The oil zones in the Adda-1 well are close to the base of the Chalk Group, suggesting fracturing due to tectonic activity.

Chalk reservoirs where no fracturing is encountered seem to be correlatable to gentle structural mounds, with low structural elevation. This type of chalk reservoir seems to be restricted to deposit zones 2, 3, and 5. We have, however, only evaluated tests performed on the G-1 and E-1 wells in this type of structures.

Flat-top chalk closures have been encountered in deposit zones 2 and 3 . These reservoirs should be

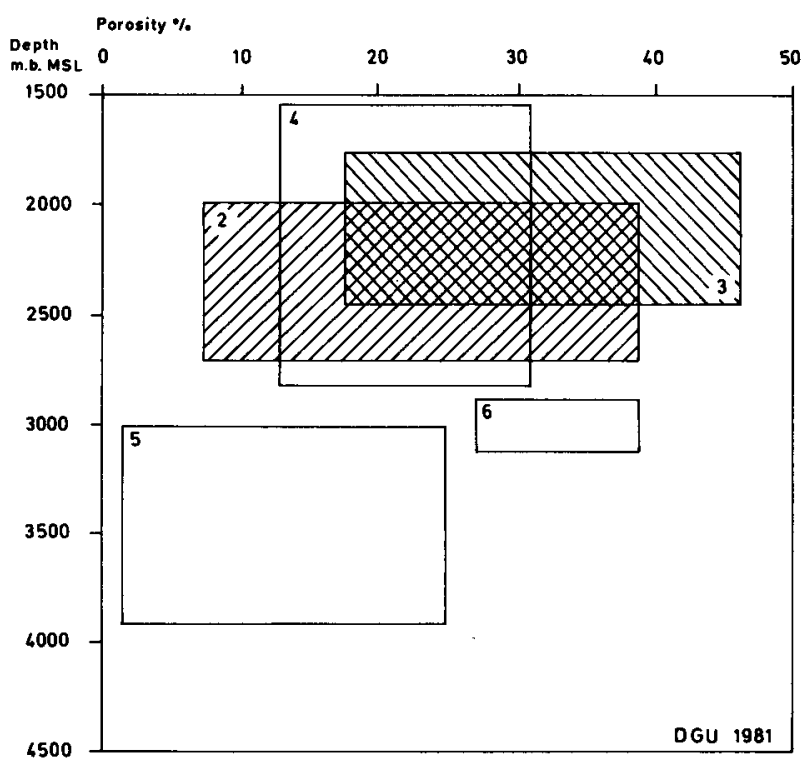

Fig. 57: Schematic presentation of porosity ranges in the Chalk Group related to Chalk deposit zones in the Central Graben. Numbers in squares refer to the individual Chalk deposit zones (cf. fig. 26). 


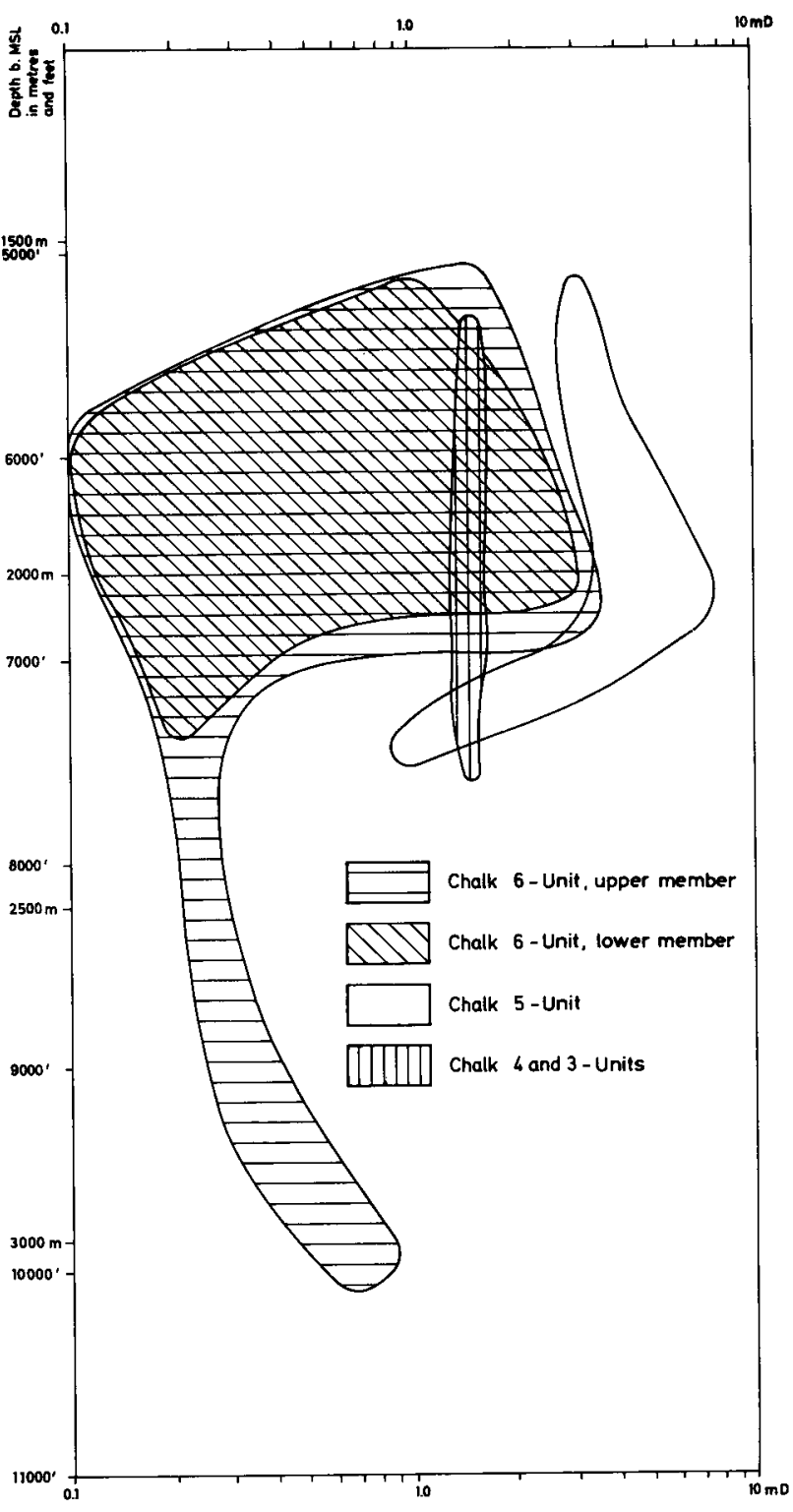

Fig. 58: Permeability distribution of Chalk Units.

expected to have a low fracture potential, provided that they follow the general pattern. It is known, as described above, that the Adda- 1 well does not follow this pattern. Core descriptions from the $\mathrm{H}-1$ well indicate fractures, but it is not known whether they contribute to permeability.

In the fracture system it is necessary to distinguish between fractures created by tension and by compression. Fractures and faults created by compression can have sealing properties, as demonstrated in the Gorm and Dan fields. Tension fractures, which are more likely to be open, may, however, later be sealed off by the precipitation of calcite cement.

Summary: Out of 16 released exploration wells in the
Chalk Group, only the O-1 well proved (though questionably) to be without hydrocarbon shows. Two of the wells found the production fields - Dan and Gorm - and production has been planned on three other fields - Tyra, Skjold, and Roar.

The Chalk Group in general can be considered as a reservoir at least down to a depth of $3100 \mathrm{~m}$. Within the Chalk Group, the upper member of the Chalk- 6 Unit and certainly the Chalk- 5 Unit can be considered to have good reservoir properties. This is based on the high primary porosity and fair permeability within these units. When naturally fractured they provide good production rates as exemplified by the Gorm field. The Chalk- 6 and Chalk-5 Units are thickest in deposit zones 3 and 4 . Through Chalk Units 4 to 1 , the primary porosity and permeability generally decreases. If naturally fractured, however, and highly oil saturated, these units have proven to produce oil at fairly high rates as seen in Adda-1 and Ruth-1. The lower member of the Chalk-6 Unit might have the poorest reservoir properties. This is caused by rather low primary porosity and permeability and also by the relatively high clay content found in this unit. When the Formation is highly naturally fractured, however, high production rates can be achieved.

Classification of chalk reservoirs: Chalk reservoirs found in the Central Graben area can be divided into three groups as follows:

1) High primary porosity and fair primary permeability. Good reservoirs of this type have thick oil zones and hydrocarbon saturation up to $95 \%$ Sh.

2) Low primary porosity and permeability. These are reasonably good reservoirs even with hydrocarbon saturation as low as $50 \%$ Sh, provided a thick oil column is present.

3) High primary porosity and fair primary permeability together with secondary fracture porosity and permeability. These are potentially the best reservoirs.

\subsection{North Sea Marl and CEN- 1 to CEN-6 Unit (Cenozoic)}

Lithologic characteristics: The units consist mainly of clastic material (clays and minor sands). The lowermost unit is dominated by a marl, upwards grading into a non-calcareous claystone or shale with subordinate layers of silt and sandstone. Above this the 
E-2

Permeability (MD).

Depth from $7000^{\circ}$ to $7200^{\circ}$ b.K.B.

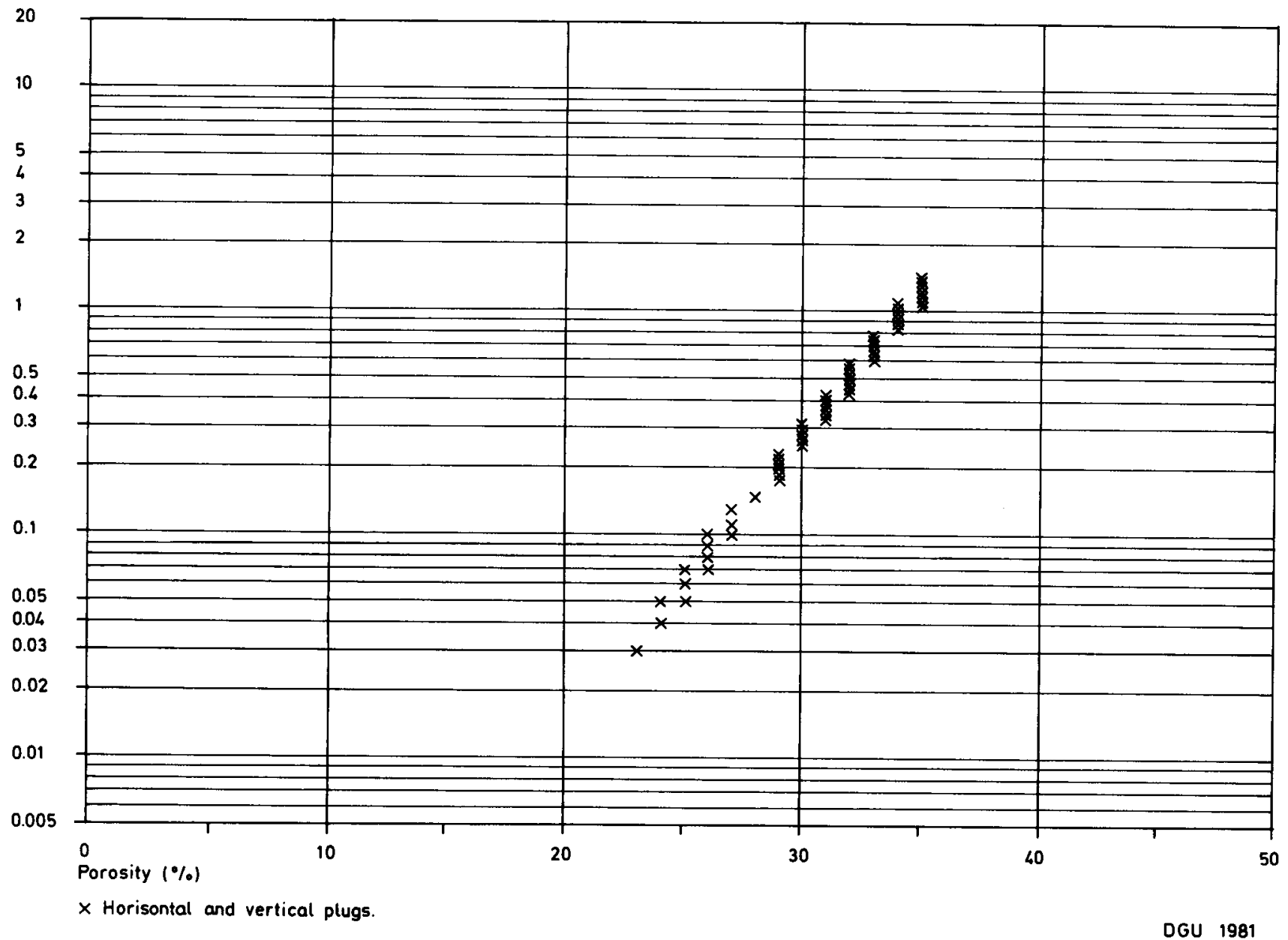

Fig. 59: Core porosity v.s. permeability plot (semi-log) from the E-2 and Ruth-1 wells. a) E-2 well. Non fractured chalk with a linear correlation. b) Ruth-1 well. Probably fractured chalk with a non-linear correlation.

sequence contains layers of volcanic tuff, which succeed into more silty claystones with subordinate interbedded limestone. Sandy intervals become more common upwards, and the uppermost part of the CEN-5 Unit is a thick gravel-containing sandstone bed. The uppermost unit consists of clay, silt- , and sandstone interbedded with lignite and shell-rich beds.

Extension, thickness, and depth: Within the Danish Central Graben the entire sequence is $1700-3100 \mathrm{~m}$ thick. The sequence thins towards the northeast and east.
Reservoir parameters: Insignificant hydrocarbon shows are found in the lower units (CEN-3, 2, 1, and North Sea Marl) where they superpose hydrocarbon reservoirs in the chalk. The hydrocarbon accumulations are thought to be formed by leakage from the chalk reservoirs.

Information is restricted to little more than a small amount of core and wire line log data. The only porosity calculation made on the North Sea Marl found an average of $30 \%$ porosity in the Adda- 1 well.

Porosity and permeability in the tuff/siltstone of the CEN-2 Unit are known from the E-1 and N-1 wells. 
RUTH - 1

Permeability (MD)

Depth from $5200^{\prime}$ to $5550^{\circ}$ b. K. B.

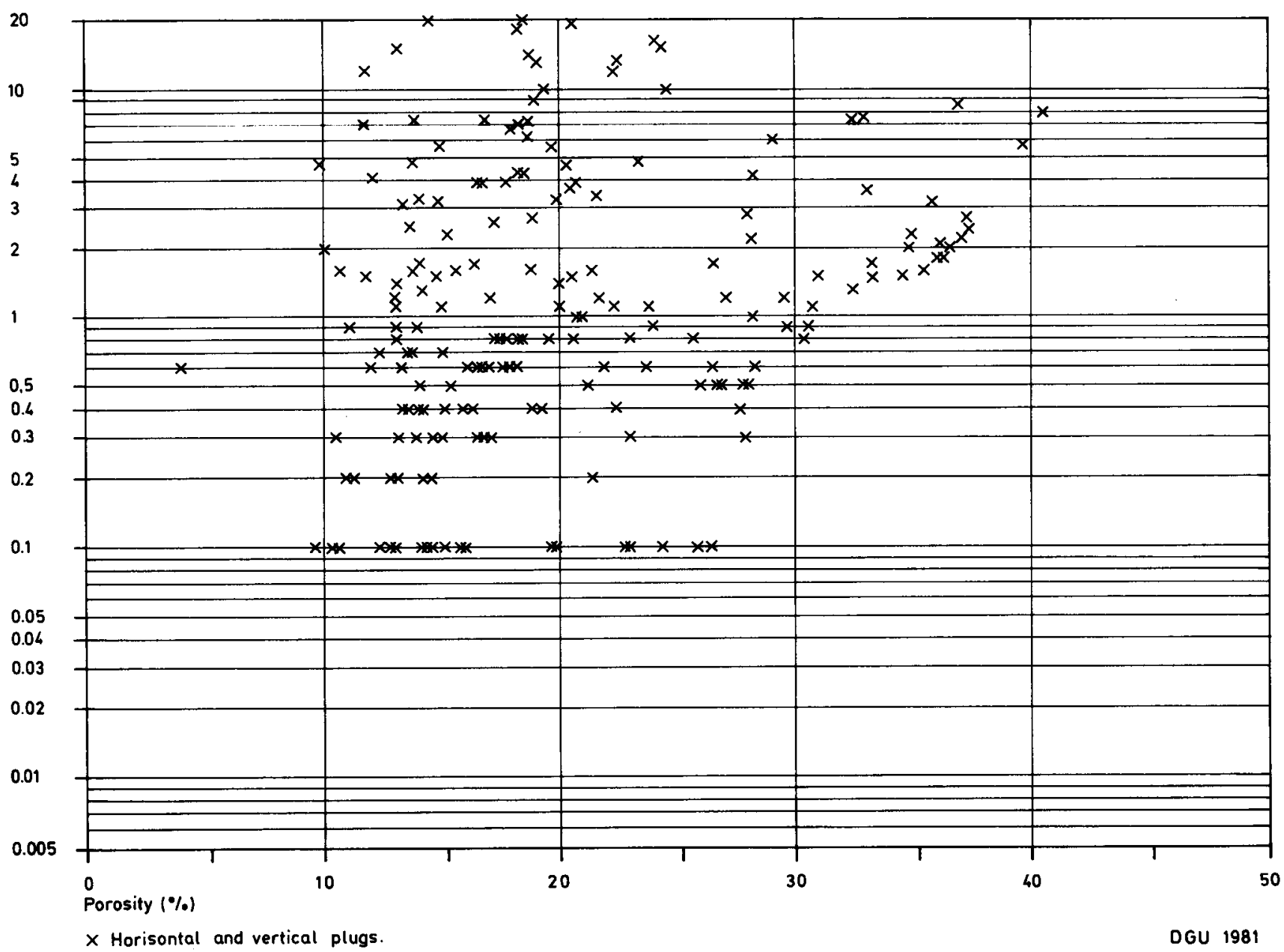

The porosity varies from $31-42 \%$ and $18-37.5 \%$ respectively. The permeability is $10 \mathrm{mD}$.

Nine limestone stringers in the CEN-3 Unit in the $\mathrm{H}-1$ well, making up $22 \mathrm{~m}$ net zone, are evaluated to have a porosity from 1 to $17 \%$, whereas the permeability is not known.
The upper part of the Cenozoic sequence becomes more sandy, and the widespread gravelly sandstone in the uppermost part of the CEN-5 Unit is correllatable from one well to another. The porosity of the sandstones ranges from $20 \%$ to $45 \%$, averaging $35 \%$. 


\subsection{Tables on the formation depth and thickness}

Legend

$\begin{array}{ll}\text { Log } & \text { Wire line logs used for identification of the boundary } \\ \text { SP } & \text { Spontaneous potential } \\ \text { Res } & \text { Resistivity } \\ \text { ITT } & \text { Interval transit time } \\ \text { GR } & \text { Gamma ray } \\ \text { FDC } & \text { Compensated formation density } \\ \text { CNL } & \text { Compensated neutron log } \\ \text { Cal } & \text { Caliper } \\ / & \text { Upper / lower boundary } \\ \text { DLL } & \text { Dual laterolog } \\ \text { TD } & \text { Total depth of the well } \\ \text { MSL } & \text { Mean sea level } \\ \text { KB } & \text { Kelly bushing } \\ & \text { Minimum thickness }\end{array}$

\section{CA-1 Unit}

\begin{tabular}{lllll} 
Well & $\begin{array}{l}\text { Depth b.KB } \\
\text { (feet) }\end{array}$ & $\begin{array}{l}\text { Log } \\
\text { (characterizing) }\end{array}$ & $\begin{array}{l}\text { Depth b.MSL } \\
\text { (m) }\end{array}$ & $\begin{array}{l}\text { Thickness } \\
\text { (m) }\end{array}$ \\
A-1 & not drilled & & \\
A-2 & not drilled & & \\
Adda-1 & not drilled & & \\
B-1 & not drilled & & \\
E-1 & not drilled & & \\
E-2 & not drilled & & \\
E-3 & not drilled & & \\
G-1 & not drilled & & \\
H-1 & not drilled & & \\
I-1 & not drilled & & \\
M-1 & not drilled & & \\
M-2 & not drilled & & \\
M-8 & not drilled & & \\
N-1 & not drilled & & \\
N-2 & not drilled & & \\
O-1 & not drilled & & \\
P-1 & 11038-11259 & & \\
Q-1 & not drilled & & \\
Ruth-1 & not drilled & & \\
T-1 & not drilled & & \\
U-1 & not drilled & & \\
V-1 & not drilled & & \\
W-1 & not drilled & & \\
\hline
\end{tabular}


Rotliegendes volcanics (with sediments)

\section{Well}

A-1

Adda-1

B-1

E-1

E-2

E-3

G-1

H-1

I-1

M-1

M-2

M-8

N-1

N-2

O-1

P-1

Q-1

Ruth-1

T-1

U-1

V-1

W-1

\section{Depth b.KB \\ (feet)}

Log

(characterizing)

not drilled

not drilled

not drilled

11841-11985

not drilled

not drilled

not drilled

not drilled

not drilled

not drilled

not drilled

not drilled

not drilled

not drilled

not drilled

not drilled

10541-11038*

14515-14745

not drilled

not drilled

not drilled

not drilled

13888-14375

Mudlog/TD
Depth b.MSL Thickness

(m)

(m)

$3574-3618$

$44^{\circ}$

$\begin{array}{lll}\text { ITT-GR/ITT-GR } & 3175-3327 & 152 \\ \text { Mudlog/TD } & 4386-4457 & 71^{\star} \\ & & \\ & & \\ \text { ITT-GR/TD } & 4199-4347 & 148^{\circ}\end{array}$

* base of volcanics and sediments of Rotliegendes

\section{Upper Rotliegendes sediments}

$\begin{array}{lllll}\text { Well } & \begin{array}{l}\text { Depth b.KB } \\ \text { (feet) }\end{array} & \begin{array}{l}\text { Log } \\ \text { (characterizing) }\end{array} & \begin{array}{l}\text { Depth b.MSL } \\ \text { (m) }\end{array} & \begin{array}{l}\text { Thickness } \\ \text { (m) }\end{array} \\ \begin{array}{l}\text { A-1 } \\ \text { A-2 }\end{array} & \begin{array}{l}\text { not drilled } \\ \text { not drilled }\end{array} & \text { Mudlog/Mudlog } & 3404-3574 & 170 \\ \text { Adda-1 } & 11283-11841 & & \\ \text { B-1 } & \text { not drilled } & & \\ \text { E-1 } & \text { not drilled } & & \\ \text { E-2 } & \text { not drilled } & & \\ \text { E-3 } & \text { not drilled } & & \\ \text { G-1 } & \text { not drilled } & & \\ \text { H-1 } & \text { not drilled } & & \\ \text { I-1 } & \text { not drilled } & & \\ \text { M-1 } & \text { not drilled } & & \\ \text { M-2 } & \text { not drilled } & & \\ \text { M-8 } & \text { not drilled } & & \\ \text { N-1 } & \text { not drilled } & & \\ \text { N-2 } & \text { not drilled } & & \\ \text { O-1 } & \text { 10353-10541 } & \text { ITT-GR/ITT-GR } & \\ \text { P-1 } & \text { absent } & & \\ \text { Q-1 } & \text { not drilled } & & \\ \text { Ruth-1 } & \text { not drilled } & & \\ \text { T-1 } & \text { not drilled } & & \\ \text { U-1 } & \text { not drilled } & & \\ \text { V-1 } & \text { absent } & & \\ \text { W-1 } & & & \\ & & & \\ & & & \\ \end{array}$




\section{Zechstein Group}

\begin{tabular}{|c|c|c|c|c|}
\hline Well & $\begin{array}{l}\text { Depth b.KB } \\
\text { (feet) }\end{array}$ & $\begin{array}{l}\text { Log } \\
\text { (characterizing) }\end{array}$ & $\begin{array}{l}\text { Depth b.MSL } \\
\text { (m) }\end{array}$ & $\begin{array}{l}\text { Thickness } \\
\text { (m) }\end{array}$ \\
\hline$A-1$ & not drilled & & & \\
\hline A-2 & not drilled & & & \\
\hline Adda-1 & not drilled & & & \\
\hline B-1 & $10282-11283$ & $\begin{array}{l}\text { Well data } \\
\text { summary sheets }\end{array}$ & $3068-3403$ & 335 \\
\hline E-1 & not drilled & & & \\
\hline E-2 & not drilled & & & \\
\hline E-3 & not drilled & & & \\
\hline G-1 & not drilled & & & \\
\hline $\mathrm{H}-1$ & not drilled & & & \\
\hline I-1 & not drilled & & & \\
\hline M-1 & not drilled & & & \\
\hline M-2 & not drilled & & & \\
\hline M-8 & not drilled & & & \\
\hline N-1 & not drilled & & & \\
\hline $\mathrm{N}-2$ & not drilled & & & \\
\hline $0-1$ & not drilled & & & \\
\hline P-1 & absent & & & \\
\hline Q-1 & $? 14385-? 14515$ & & $4347-4386$ & 39 \\
\hline Ruth-1 & $5590-5618$ & Mudlog/TD & $1662-1677$ & $8^{6}$ \\
\hline T-1 & $7686-8713$ & $\begin{array}{l}\text { Well data } \\
\text { summary sheets/TD }\end{array}$ & $2318-2631$ & $312^{6}$ \\
\hline U-1 & not drilled & & & \\
\hline V-1 & not drilled & & & \\
\hline W-1 & $? 13860-? 12888$ & & $4190-4199$ & 9 \\
\hline
\end{tabular}

\section{Bacton Group (Bunter Sandstone and Shale Formations) \\ Well \\ Depth b.KB
(feet) \\ Log \\ (characterizing) \\ Depth b.MSL \\ (m) \\ Thickness \\ (m)}

A-1

A-2

Adda-1

B-1

E-1

E-2

E-3

G-1

H-1

I-1

M-1

M-2

M-8

N-1

N-2

O-1

P-1

Q-1

Ruth-1

T-1

U-1

V-1

W-1 not drilled

not drilled

not drilled

9990-10190

not drilled

not drilled

not drilled

not drilled

not drilled

not drilled

not drilled

not drilled

not drilled

not drilled

not drilled

not drilled

absent

13910-14385

not drilled

not drilled

14651-16045

not drilled

absent
CNL-GR/CNL-GR $\quad 3009-3060 \quad 51$
ITT-GR/ITT-GR $\quad 4203-4348 \quad 145$

$\begin{array}{lll}\text { ITT-GR/TD } & 4437-4962 & 425^{\circ}\end{array}$ 
Dowsing Dolomitic Formation

\begin{tabular}{|c|c|c|c|c|}
\hline Well & $\begin{array}{l}\text { Depth b.KB } \\
\text { (feet) }\end{array}$ & $\begin{array}{l}\text { Log } \\
\text { (characterizing) }\end{array}$ & $\begin{array}{l}\text { Depth b.MSL } \\
\text { (m) }\end{array}$ & $\begin{array}{l}\text { Thickness } \\
\text { (m) }\end{array}$ \\
\hline A-1 & not drilled & & & \\
\hline A-2 & $10050-11143$ & Mudlog/TD & $3027-3360$ & 333 \\
\hline Adda-1 & not drilled & & & \\
\hline B-1 & absent & & & \\
\hline E-1 & not drilled & & & \\
\hline E-2 & not drilled & & & \\
\hline E-3 & not drilled & & & \\
\hline G-1 & not drilled & & & \\
\hline H-1 & not drilled & & & \\
\hline I-1 & not drilled & & & \\
\hline M-1 & not drilled & & & \\
\hline M-2 & not drilled & & & \\
\hline M-8 & not drilled & & & \\
\hline $\mathrm{N}-1$ & not drilled & & & \\
\hline $\mathrm{N}-2$ & not drilled & & & \\
\hline O-1 & not drilled & & & \\
\hline P-1 & absent & & & \\
\hline Q-1 & absent & & & \\
\hline Ruth-1 & not drilled & & & \\
\hline T-1 & not drilled & & & \\
\hline U-1 & $13074-14651$ & ITT-GR/ITT-GR & $3957-4437$ & 480 \\
\hline V-1 & $12080-12654$ & ITT-GR/TD & $3648-3823$ & $175^{\circ}$ \\
\hline W-1 & absent & & & \\
\hline
\end{tabular}

\section{Dudgeon Saliferous Formation}

Well

A-1

A-2

Adda-1

B-1

E-1

E-2

E-3

G-1

H-1

I-1

M-1

M-2

M-8

$\mathrm{N}-1$

$\mathrm{N}-2$

O-1

P-1

Q-1

Ruth-1

T-1

U-1

V-1

W-1
Depth b.KB

(feet)

(characterizing)

not drilled

absent

not drilled

absent

not drilled

not drilled

not drilled

not drilled

not drilled

not drilled

not drilled

not drilled

11559-12007

not drilled

not drilled

11193-11740

absent

absent

not drilled

not drilled

12159-13074

absent

absent
ITT-GR/TD

ITT-GR/TD

ITT-GR/ITT-GR
Depth b.MSL Thickness

(m)

(m)
$3494-3647 \quad 153^{\circ}$

$3384-3550 \quad 166^{\circ}$ 
Triton Anhydritic Formation

\begin{tabular}{|c|c|c|c|c|}
\hline Well & $\begin{array}{l}\text { Depth b.KB } \\
\text { (feet) }\end{array}$ & $\begin{array}{l}\text { Log } \\
\text { (characterizing) }\end{array}$ & $\begin{array}{l}\text { Depth b.MSL } \\
\text { (m) }\end{array}$ & $\begin{array}{l}\text { Thickness } \\
\text { (m) }\end{array}$ \\
\hline A-1 & not drilled & & & \\
\hline A-2 & absent & & & \\
\hline Adda-1 & not drilled & & & \\
\hline B-1 & absent & & & \\
\hline E-1 & not drilled & & & \\
\hline E-2 & not drilled & & & \\
\hline E-3 & not drilled & & & \\
\hline G-1 & not drilled & & & \\
\hline H-1 & not drilled & & & \\
\hline I-1 & not drilled & & & \\
\hline M-1 & not drilled & & & \\
\hline M-2 & not drilled & & & \\
\hline M-8 & $10793-11559$ & ITT-GR/ITT-GR & $3260-3494$ & 234 \\
\hline $\mathrm{N}-1$ & not drilled & & & \\
\hline $\mathrm{N}-2$ & not drilled & & & \\
\hline $0-1$ & $10401-11193$ & ITT-GR/ITT-GR & $3142-3384$ & 242 \\
\hline P-1 & absent & & & \\
\hline Q-1 & absent & & & \\
\hline Ruth-1 & not drilled & & & \\
\hline T-1 & not drilled & & & \\
\hline U-1 & $11250-12159$ & ITT-GR/ITT-GR & $3401-3678$ & 277 \\
\hline$V-1$ & absent & & & \\
\hline W-1 & absent & & & \\
\hline
\end{tabular}

\section{Winterton Formation}

\begin{tabular}{|c|c|c|c|c|}
\hline Well & $\begin{array}{l}\text { Depth b.KB } \\
\text { (feet) }\end{array}$ & $\begin{array}{l}\text { Log } \\
\text { (characterizing) }\end{array}$ & $\begin{array}{l}\text { Depth b.MSL } \\
\text { (m) }\end{array}$ & $\begin{array}{l}\text { Thickness } \\
\text { (m) }\end{array}$ \\
\hline$A-1$ & not drilled & & & \\
\hline$A-2$ & absent & & & \\
\hline Adda-1 & not drilled & & & \\
\hline B-1 & absent & & & \\
\hline E-1 & not drilled & & & \\
\hline E-2 & not drilled & & & \\
\hline E-3 & not drilled & & & \\
\hline G-1 & not drilled & & & \\
\hline $\mathrm{H}-1$ & not drilled & & & \\
\hline I-1 & not drilled & & & \\
\hline M-1 & not drilled & & & \\
\hline M-2 & not drilled & & & \\
\hline M-8 & $10771-10793$ & ITT-GR/ITT-GR & $3253-3260$ & 7 \\
\hline $\mathrm{N}-1$ & not drilled & & & \\
\hline $\mathrm{N}-2$ & not drilled & & & \\
\hline $0-1$ & $10347-10401$ & ITT-GR/ITT-GR & $3126-3142$ & 16 \\
\hline P-1 & absent & & & \\
\hline Q-1 & absent & & & \\
\hline Ruth-1 & not drilled & & & \\
\hline T-1 & not drilled & & & \\
\hline $\mathrm{U}-1$ & $11209-11250$ & ITT-GR/ITT-GR & $3389-3401$ & 12 \\
\hline$V-1$ & absent & & & \\
\hline W-1 & absent & & & \\
\hline
\end{tabular}




\section{Fjerritslev Formation}

\begin{tabular}{|c|c|c|c|c|}
\hline Well & $\begin{array}{l}\text { Depth b.KB } \\
\text { (feet) }\end{array}$ & $\begin{array}{l}\text { Log } \\
\text { (characterizing) }\end{array}$ & $\begin{array}{l}\text { Depth b.MSL } \\
\text { (m) }\end{array}$ & $\begin{array}{l}\text { Thickness } \\
\text { (m) }\end{array}$ \\
\hline A-1 & not drilled & & & \\
\hline A-2 & absent & & & \\
\hline Adda-1 & not drilled & & & \\
\hline B-1 & no evaluation & & & \\
\hline E-1 & not drilled & & & \\
\hline E-2 & not drilled & & & \\
\hline E-3 & not drilled & & & \\
\hline G-1 & not drilled & & & \\
\hline H-1 & not drilled & & & \\
\hline I-1 & not drilled & & & \\
\hline M-1 & not drilled & & & \\
\hline M-2 & not drilled & & & \\
\hline M-8 & $10467-10771$ & ITT-GR/ITT-GR & $3161-3154$ & 93 \\
\hline$N-1$ & not drilled & & & \\
\hline $\mathrm{N}-2$ & not drilled & & & \\
\hline O-1 & $9838-10347$ & ITT-GR/ITT-GR & $2971-3126$ & 155 \\
\hline P. 1 & absent & & & \\
\hline Q-1 & $13608-13910$ & ITT-GR/ITT-GR & $4111-4203$ & 92 \\
\hline Ruth-1 & not drilled & & & \\
\hline T-1 & not drilled & & & \\
\hline U-1 & $11048-11209$ & ITT-GR/ITT-GR & $3340-3389$ & 49 \\
\hline V-1 & absent & & & \\
\hline W-1 & absent & & & \\
\hline
\end{tabular}

\section{J-2 Unit, all members}

\begin{tabular}{|c|c|c|c|c|}
\hline Well & $\begin{array}{l}\text { Depth b.KB } \\
\text { (feet) }\end{array}$ & $\begin{array}{l}\text { Log } \\
\text { (characterizing) }\end{array}$ & $\begin{array}{l}\text { Depth b.MSL } \\
\text { (m) }\end{array}$ & $\begin{array}{l}\text { Thickness } \\
\text { (m) }\end{array}$ \\
\hline A-1 & not drilled & & & \\
\hline A-2 & $9920-10050$ & Mud log & $2988-3027$ & 39 \\
\hline Adda-1 & not drilled & & & \\
\hline B-1 & no evaluation & & & \\
\hline E-1 & not drilled & & & \\
\hline E-2 & not drilled & & & \\
\hline E-3 & not drilled & & & \\
\hline G-1 & not drilled & & & \\
\hline H-1 & not drilled & & & \\
\hline I-1 & not drilled & & & \\
\hline M-1 & not drilled & & & \\
\hline M-2 & not drilled & & & \\
\hline M-8 & $10143-10467$ & ITT-GR/ITT-GR & $3062-3161$ & 99 \\
\hline N-1 & not drilled & & & \\
\hline $\mathrm{N}-2$ & not drilled & & & \\
\hline O-1 & $8901-9840$ & ITT-GR/ITT & $2685-2971$ & 286 \\
\hline P-1 & absent & & & \\
\hline Q-1 & $13429-13608$ & ITT-GR/ITT-GR & $4063-4111$ & 48 \\
\hline Ruth-1 & not drilled & & & \\
\hline $\mathrm{T}-1$ & not drilled & & & \\
\hline $\mathrm{U}-1$ & $10665-11048$ & ITT-GR/CNL-FDC & $3222-3339$ & 117 \\
\hline V-1 & absent & & & \\
\hline W-1 & absent & & & \\
\hline
\end{tabular}


J-2 Unit, upper member

\begin{tabular}{|c|c|c|c|c|}
\hline Well & $\begin{array}{l}\text { Depth b.KB } \\
\text { (feet) }\end{array}$ & $\begin{array}{l}\text { Log } \\
\text { (characterizing) }\end{array}$ & $\begin{array}{l}\text { Depth b.MSL } \\
\text { (m) }\end{array}$ & $\begin{array}{l}\text { Thickness } \\
\text { (m) }\end{array}$ \\
\hline$A-1$ & not drilled & & & \\
\hline A-2 & $9920-9970$ & Mud Log & $2988-3003$ & 15 \\
\hline Adda-1 & not drilled & & & \\
\hline B-1 & no evaluation & & & \\
\hline E-1 & not drilled & & & \\
\hline E-2 & not drilled & & & \\
\hline E-3 & not drilled & & & \\
\hline G-1 & not drilled & & & \\
\hline H-1 & not drilled & & & \\
\hline I-1 & not drilled & & & \\
\hline M-1 & not drilled & & & \\
\hline M-2 & not drilled & & & \\
\hline M-8 & $10143-10260$ & ITT/GR-ITT & $3062-3098$ & 36 \\
\hline $\mathrm{N}-1$ & not drilled & & & \\
\hline $\mathrm{N}-2$ & not drilled & & & \\
\hline O-1 & $8901-9425$ & ITT-GR/ITT-GR & $2685-2845$ & 160 \\
\hline P-1 & absent & & & \\
\hline Q-1* & $?-?$ & $?-?$ & $?$ & \\
\hline Ruth-1 & not drilled & & & \\
\hline $\mathrm{T}-1$ & not drilled & & & \\
\hline U-1 & $10665-10853$ & ITT-GR/ITT-GR & $3222-3280$ & 58 \\
\hline$V-1$ & absent & & & \\
\hline W-1 & absent & & & \\
\hline
\end{tabular}

\section{J-2 Unit, lower member}

\begin{tabular}{|c|c|c|c|c|}
\hline Well & $\begin{array}{l}\text { Depth b.KB } \\
\text { (feet) }\end{array}$ & $\begin{array}{l}\text { Log } \\
\text { (characterizing) }\end{array}$ & $\begin{array}{l}\text { Depth b.MSL } \\
\text { (m) }\end{array}$ & $\begin{array}{l}\text { Thickness } \\
\text { (m) }\end{array}$ \\
\hline A-1 & not drilled & & & \\
\hline A-2 & $9970-10050$ & Mud Log & $3003-3027$ & 24 \\
\hline Adda-1 & not drilled & & & \\
\hline B-1 & no evaluation & & & \\
\hline E-1 & not drilled & & & \\
\hline $\mathrm{E}-2$ & not drilled & & & \\
\hline E-3 & not drilled & & & \\
\hline G-1 & not drilled & & & \\
\hline H-1 & not drilled & & & \\
\hline I-1 & not drilled & & & \\
\hline M-1 & not drilled & & & \\
\hline M-2 & not drilled & & & \\
\hline M-8 & $10260-10467$ & ITT-GR/ITT-GR & $3098-3161$ & 37 \\
\hline $\mathrm{N}-1$ & not drilled & & & \\
\hline N-2 & not drilled & & & \\
\hline O-1 & $9425-9838$ & ITT-GR/ITT & $2845-2971$ & 126 \\
\hline P-1 & absent & & & \\
\hline Q-1* & $?-?$ & & $?-?$ & $?$ \\
\hline Ruth-1 & not drilled & & & \\
\hline $\mathrm{T}-1$ & not drilled & & & \\
\hline $\mathrm{U}-1$ & $10853-11048$ & ITT-GR/FDC-CNL & $3280-3339$ & 41 \\
\hline V-1 & absent & & & \\
\hline W-1 & absent & & & \\
\hline
\end{tabular}




\section{W-1 Unit}

Well

A-1

A-2

Adda-1

B-1

E-1

E-2

E-3

G-1

H-1

I-1

M-1

M-2

M-8

N-1

$\mathrm{N}-2$

O-1

P-1

Q-1

Ruth-1

T-1

U-1

V-1

W-1

\section{J-3 Unit}

Well

A-1

A-2

Adda-1

B-1

E-1

E-2

E-3

G-1

H-1

I-1

M-1

M-2

M-8

N-1

N-2

O-1

P-1

Q-1

Ruth-1

T-1

U-1

V-1

W-1
Depth b.KB

(feet)

Log

(characterizing)

not drilled

absent

not drilled

no evaluation

not drilled

not drilled

not drilled

absent

not drilled

not drilled

not drilled

not drilled

absent

not drilled

not drilled

absent

absent

absent

not drilled

not drilled

absent

absent

13521-13860

ITT-GR/ITT
Depth b.MSL

(m)

Thickness

(m)
$4087-4184$

97

Depth b.MSL Thickness

(m)

(m)

(feet)

(characterizing)

Mudlog

$?-2988$

? - 9920

not drilled

no evaluation

not drilled

not drilled

not drilled

12037-12517

not drilled

not drilled

not drilled

not drilled

8940-10143

not drilled

not drilled

? - 8901

absent

not drilled

not drilled

not drilled

9595-10665

?10601-12080

ITT-GR/TD

$3632-3778$

$146^{\circ}$

absent

$\begin{array}{rcc}\text { ITT-GR/ITT-GR } & 2695-3062 & 367 \\ \text { /ITT-GR } & ?-2685 & ? \\ & & \\ & & \\ & & 326 \\ \text { ITT-GR/ITT-GR } & 2896-3222 & ? 450 \\ \text { ITT-GR/ITT-GR } & ? 3198-3648 & \end{array}$




\section{J-4 Unit}

\begin{tabular}{|c|c|c|c|c|}
\hline Well & $\begin{array}{l}\text { Depth b.KB } \\
\text { (feet) }\end{array}$ & $\begin{array}{l}\text { Log } \\
\text { (characterizing) }\end{array}$ & $\begin{array}{l}\text { Depth b.MSL } \\
\text { (m) }\end{array}$ & $\begin{array}{l}\text { Thickness } \\
\text { (m) }\end{array}$ \\
\hline A-1 & not drilled & & & \\
\hline A-2 & 7470-? & Mudlog & 2241-? & $?$ \\
\hline Adda-1 & $8400-10005$ & ITT-GR/TD log & 2526- 3015 & $489^{\circ}$ \\
\hline B-1* & $?-?$ & & $?-?$ & $?$ \\
\hline E-1 & $9727-13403$ & ITT-GR/TD log & $2928-4048$ & $1120^{\circ}$ \\
\hline E-2 & not drilled & & & \\
\hline E-3 & not drilled & & & \\
\hline G-1 & 8088- 12037 & ITT-GR/ITT-GR & 2429- 3632 & 1204 \\
\hline H-1 & not drilled & & & \\
\hline I-1 & $11018-12848$ & ITT-GR/TD log & 3321- 3879 & $558^{\circ}$ \\
\hline M-1 & $7190-7374$ & ITT-GR/TD log & 2158- 2475 & $117^{\star}$ \\
\hline M-2 & not drilled & & & \\
\hline M-8 & 7517- 8940 & ITT-GR/ITT-GR & 2262- 2695 & 433 \\
\hline $\mathrm{N}-1$ & not drilled & & & \\
\hline $\mathrm{N}-2$ & not drilled & & & \\
\hline $0-1^{*}$ & $?-?$ & & $?-?$ & $?$ \\
\hline P-1 & absent & & & \\
\hline Q-1* & $?-?$ & & $?-?$ & $?$ \\
\hline Ruth-1 & not drilled & & & \\
\hline $\mathrm{T}-1$ & not drilled & & & \\
\hline $\mathrm{U}-1$ & $8190-9595$ & ITT-GR/ITT-GR & 2468- 2896 & 428 \\
\hline$V-1$ & $9462-? 10601$ & ITT-GR/ITT-GR & 2833-?3198 & $? 347$ \\
\hline W-1 & $12333-13521$ & ITT-GR/ITT-GR & $3725-4087$ & 362 \\
\hline
\end{tabular}

\section{LC-1 Unit}

\begin{tabular}{|c|c|c|c|c|}
\hline Well & $\begin{array}{l}\text { Depth b.KB } \\
\text { (feet) }\end{array}$ & $\begin{array}{l}\text { Log } \\
\text { (characterizing) }\end{array}$ & $\begin{array}{l}\text { Depth b.MSL } \\
\text { (m) }\end{array}$ & $\begin{array}{l}\text { Thickness } \\
\text { (m) }\end{array}$ \\
\hline A-1 & not drilled & & & \\
\hline A-2 & absent & & & \\
\hline Adda-1 & absent & & & \\
\hline B-1 & no evaluation & & & \\
\hline E-1 & absent & & & \\
\hline E-2 & not drilled & & & \\
\hline E-3 & not drilled & & & \\
\hline G-1 & absent & & & \\
\hline H-1 & not drilled & & & \\
\hline I-1 & absent & & & \\
\hline M-1 & absent & & & \\
\hline M-2 & not drilled & & & \\
\hline M-8 & absent & & & \\
\hline $\mathrm{N}-1$ & not drilled & & & \\
\hline $\mathrm{N}-2$ & not drilled & & & \\
\hline O-1 & absent & & & \\
\hline P-1 & absent & & & \\
\hline Per-1 & absent & & & \\
\hline Q-1 & absent & & & \\
\hline Ruth-1 & absent & & & \\
\hline$T-1$ & no evaluation & & & \\
\hline $\mathrm{U}-1$ & absent & & & \\
\hline$V-1$ & $8972-9462$ & $\begin{array}{l}\text { GR,FDC,CNL,ITT/ } \\
\text { GR,FDC,CNL,ITT }\end{array}$ & $2702-2851$ & 149 \\
\hline
\end{tabular}


Valhall Formation

\begin{tabular}{|c|c|c|c|c|}
\hline Well & $\begin{array}{l}\text { Depth b.KB } \\
\text { (feet) }\end{array}$ & $\begin{array}{l}\text { Log } \\
\text { (characterizing) }\end{array}$ & $\begin{array}{l}\text { Depth b.MSL } \\
\text { (m) }\end{array}$ & $\begin{array}{l}\text { Thickness } \\
\text { (m) }\end{array}$ \\
\hline A-1 & not drilled & & & \\
\hline A-2 & $7260-7470$ & Mudlog/Mudlog & $2177-2241$ & 64 \\
\hline Adda-1 & $7530-8400$ & ITT-GR/ITT-GR & $2262-2526$ & 264 \\
\hline B-1 & no evaluation & & & \\
\hline E-1 & 8297- 9727 & ITT-GR/ITT-GR & $2492-2928$ & 236 \\
\hline E-2 & not drilled & & & \\
\hline E-3 & $8350-8722$ & GR/GR & $2515-2628$ & $113^{\circ}$ \\
\hline G-1 & $7410-8088$ & ITT-GR/ITT-GR & $2222-2429$ & 207 \\
\hline $\mathrm{H}-1$ & no evaluation & & & \\
\hline I-1 & $9508-11018$ & ITT-GR/ITT-GR & $2862-3321$ & 459 \\
\hline M-1 & $6902-7190$ & ITT-GR/ITT-GR & 2071-2159 & 88 \\
\hline M-2 & not drilled & & & \\
\hline M-7 & $8934-9070$ & Mudlog/Mudlog & $2691-2733$ & $42^{\circ}$ \\
\hline M-8 & $7280-7517$ & ITT-GR/ITT-GR & $2190-2262$ & 72 \\
\hline $\mathrm{N}-1$ & not drilled & & & \\
\hline $\mathrm{N}-2$ & not drilled & & & \\
\hline O-1 & 7580-? & CNL-GR/? & 2283-? & \\
\hline P-1 & absent & & & \\
\hline Per-1 & absent & & & \\
\hline \multicolumn{5}{|l|}{ Q-1 ? } \\
\hline Ruth-1 & absent & & & \\
\hline $\mathrm{T}-1$ & no evaluation & & & \\
\hline U-1 & $8112-8190$ & ITT-GR/ITT-GR & $2445-2468$ & 23 \\
\hline \multirow[t]{2}{*}{ V-1 } & $8937-8972$ & GR-ITT/ & $2691-2702$ & 12 \\
\hline & & GR,GDC,CNL,ITT & & \\
\hline W-1 & ? & & & \\
\hline
\end{tabular}

\section{Rødby Formation}

\begin{tabular}{|c|c|c|c|c|}
\hline Well & $\begin{array}{l}\text { Depth b.KB } \\
\text { (feet) }\end{array}$ & $\begin{array}{l}\text { Log } \\
\text { (characterizing) }\end{array}$ & $\begin{array}{l}\text { Depth b.MSL } \\
\text { (m) }\end{array}$ & $\begin{array}{l}\text { Thickness } \\
\text { (m) }\end{array}$ \\
\hline A-1 & not drilled & & & \\
\hline A-2 & $7240-7260$ & Mudlog/Mudlog & $2171-2177$ & 6 \\
\hline Adda-1 & $7512-7530$ & ITT-GR/ITT-GR & $2256-2262$ & 6 \\
\hline B-1 & no evaluation & & & \\
\hline E-1 & $8162-8297$ & ITT-GR/ITT-GR & $2451-2492$ & 41 \\
\hline E-2 & not drilled & & & \\
\hline E-3 & $8305-8350$ & ITT-GR/GR & $2502-2515$ & 13 \\
\hline G-1 & absent & & & \\
\hline H-1 & not drilled & & & \\
\hline I-1 & $9355-9508$ & ITT-GR/ITT-GR & $2815-2862$ & 47 \\
\hline M-1 & $6858-6902$ & ITT-GR/ITT-GR & $2057-2071$ & 14 \\
\hline M-2 & not drilled & & & \\
\hline M-8 & $7212-7280$ & ITT-GR/ITT-GR & $2169-2190$ & 21 \\
\hline $\mathrm{N}-1$ & not drilled & & & \\
\hline $\mathrm{N}-2$ & not drilled & & & \\
\hline $0-1$ & $7570-7580$ & CNL-GR/CNL-GR & $2280-2283$ & 3 \\
\hline P-1 & absent & & & \\
\hline Per-1 & absent & & & \\
\hline Q-1 & no evaluation & & & \\
\hline Ruth-1 & absent & & & \\
\hline$T-1$ & no evaluation & & & \\
\hline U-1 & absent & & & \\
\hline V-1 & absent & & & \\
\hline W-1 & absent & & & \\
\hline
\end{tabular}




\section{Chalk-1 Unit}

\begin{tabular}{|c|c|c|c|c|}
\hline Well & $\begin{array}{l}\text { Depth b.KB } \\
\text { (feet) }\end{array}$ & $\begin{array}{l}\text { Log } \\
\text { (characterizing) }\end{array}$ & $\begin{array}{l}\text { Depth b.MSL } \\
\text { (m) }\end{array}$ & $\begin{array}{l}\text { Thickness } \\
\text { (m) }\end{array}$ \\
\hline A-1 & no logs & & & \\
\hline A-2 & no logs & & & \\
\hline Adda-1 & $7420-7512$ & ITT-GR/ITT-GR & $2227-2256$ & 28 \\
\hline B-1 & absent & & & \\
\hline E-1 & $8115-8162$ & ITT-GR/ITT-GR & $2436-2451$ & 14 \\
\hline E-2 & absent & & & \\
\hline E-3 & absent & & & \\
\hline G-1 & no logs & & & \\
\hline $\mathrm{H}-1$ & absent & & & \\
\hline I-1 & absent & & & \\
\hline M-1 & absent & & & \\
\hline $\mathbf{M}-2$ & no evaluation & & & \\
\hline M-8 & absent & & & \\
\hline $\mathrm{N}-1$ & not drilled & & & \\
\hline $\mathrm{N}-2$ & no evaluation & & & \\
\hline O-1 & $7500-7580$ & ITT-GR/ITT-GR & $2258-2282$ & 24 \\
\hline P-1 & absent & & & \\
\hline Q-1 & ?no logs* & & & \\
\hline Ruth-1 & absent & & & \\
\hline T-1 & absent & & & \\
\hline U-1 & absent & & & \\
\hline V-1 & absent & & & \\
\hline W-1 & absent & & & \\
\hline
\end{tabular}

\section{Chalk-2 Unit}

\begin{tabular}{|c|c|c|c|c|}
\hline Well & $\begin{array}{l}\text { Depth b.KB } \\
\text { (feet) }\end{array}$ & $\begin{array}{l}\text { Log } \\
\text { (characterizing) }\end{array}$ & $\begin{array}{l}\text { Depth b.MSL } \\
\text { (m) }\end{array}$ & $\begin{array}{l}\text { Thickness } \\
\text { (m) }\end{array}$ \\
\hline A-1 & no logs & & & \\
\hline A-2 & no logs & & & \\
\hline Adda-1 & $7138-7420$ & ITT-GR/ITT-GR & $2142-2227$ & 85 \\
\hline B-1 & $7520-7595$ & ITT-GR/ITT-GR & $2256-2279$ & 23 \\
\hline E-1 & $7500-8115$ & ITT-GR/ITT-GR & $2249-2436$ & 187 \\
\hline E-2 & no evaluation & & & \\
\hline E-3 & no evaluation & & & \\
\hline G-1 & $7120-7410$ & ITT-GR/ITT-GR & $2133-2222$ & 89 \\
\hline H-1 & absent & & & \\
\hline I-1 & absent & & & \\
\hline M-1 & $6590-6858$ & ITT-GR/ITT-GR & $1975-2057$ & $82 ?$ \\
\hline M-2 & no evaluation & & & \\
\hline M-8 & $6880-7212$ & ITT-GR/ITT-GR & $2067-2169$ & 102 \\
\hline $\mathrm{N}-1$ & 7904-TD 8155 & ITT-GR/ITT-GR & $2377-2453$ & $76^{\circ}$ \\
\hline $\mathrm{N}-2$ & no evaluation & & & \\
\hline O-1 & $7408-7500$ & ITT-GR/ITT-GR & $2230-2258$ & 28 \\
\hline P-1 & absent & & & \\
\hline Q-1 & $12128-13000$ & ITT-GR/ITT-GR & $3659-3925$ & 266 \\
\hline Ruth-1 & absent & & & \\
\hline $\mathrm{T}-1$ & absent & & & \\
\hline $\mathrm{U}-1$ & 7898- 8109 & ITT-GR/ITT-GR & $2379-2443$ & 64 \\
\hline V-1 & $8375-8937$ & ITT-GR/ITT-GR & $2519-2690$ & 171 \\
\hline W-1 & $11782-12333$ & ITT-GR/ITT-GR & $3557-3725$ & 168 \\
\hline
\end{tabular}


Chalk-3 Unit

Well

A-1

A-2

Adda-1

B-1

E-1

E-2

E-3

G-1

H-1

I-1

M-1

M-2

M-8

N-1

$\mathrm{N}-2$

O-1

P-1

Q-1

Ruth-1

T-1

U-1

V-1

W-1

$$
\begin{aligned}
& \text { Depth b.KB } \\
& \text { (feet) }
\end{aligned}
$$

no logs

no logs

7063- 7138

7447- 7520

7325- 7500

no evaluation

no evaluation

6875- 7120

absent

absent

6471- 6590

no evaluation

6681- 6880

7745- 7904

no evaluation

7349- 7408

absent

11640-12128

absent

absent

7800- 7898

8025- 8375

11267-11782
Log

(characterizing)
Depth b.MSL

(m)

Thickness

(m)

$\begin{array}{lll}\text { ITT-GR/ITT-GR } & 2119-2142 & 23 \\ \text { ITT-GR/ITT-GR } & 2234-2256 & 22 \\ \text { ITT-GR/ITT-GR } & 2195-2249 & 54 \\ & & \\ \text { ITT-GR/ITT-GR } & 2067-2142 & 75 \\ & & \\ \text { ITT-GR/ITT-GR } & 1939-1975 & ? 36 \\ & & \\ \text { ITT-GR/ITT-GR } & 2007-2067 & 60 \\ \text { ITT-GR/ITT-GR } & 2328-2377 & 49 \\ & & \\ \text { ITT-GR/ITT-GR } & 2212-2230 & ? 18 \\ & & \\ \text { ITT-GR/ITT-GR } & 3510-3659 & 149 \\ & & \\ \text { ITT-GR/ITT-GR } & 2339-2369 & 30 \\ \text { ITT-GR/ITT-GR } & 2412-2519 & 107 \\ & 3400-3557 & 157\end{array}$

Chalk-4 Unit

Well

A-1

A-2

Adda-1

B-1

E-1

E-2

E-3

G-1

H-1

I-1

M-1

M-2

M-8

N-1

$\mathrm{N}-2$

$\mathrm{O}-1$

P-1

Q-1

Ruth-1

T-1

U-1

V-1

W-1

* (6650') base log.
Depth b.KB

(feet)

6262-(6650)*

absent

7402- 7447

7072- 7325

no evaluation

no evaluation

6830- 6875

6981-TD 7100

9241- 9355

6118- 6471

no evaluation

6256- 6681

7147- 7745

no evaluation

7187- 7349

9865-10350

10730-11640

5290- 5586

7519- 7685

7594- 7800

7639- 8025

10695-11267
Log

(characterizing)

ITT-GR/ITT-GR

ITT-GR/ITT-GR

ITT-GR/ITT-GR

ITT-GR/ITT-GR

ITT-GR/ITT-GR

ITT-GR/ITT-GR

ITT-GR/ITT-GR

ITT-GR/ITT-GR

ITT-GR/ITT-GR

ITT-GR/ITT-GR

ITT-GR/ITT-GR

ITT-GR/ITT-GR

ITT-GR/ITT-GR

ITT-GR/ITT-GR

ITT-GR/ITT-GR

ITT-GR/ITT-GR

ITT-GR/ITT-GR
Depth b.MSL Thickness

(m)

(m)

1873-1991

$118^{\circ}$

2220-2234 14

2118-2195 77

2045-2058 13

2091-2127 36

2779-2814 35

1831-1935 108

1877-2007 130

2146-2328 $\quad 182$

2163-2212 49

2969-3117 $\quad 148$

3233-3510 277

1577-1668 91

$2267-2317 \quad 50$

2286-2349 63

2295-2412 117

3226-3400 $\quad 174$ 
Chalk-5 Unit

\begin{tabular}{|c|c|c|c|c|}
\hline Well & $\begin{array}{l}\text { Depth b.KB } \\
\text { (feet) }\end{array}$ & $\begin{array}{l}\text { Log } \\
\text { (characterizing) }\end{array}$ & $\begin{array}{l}\text { Depth b.MSL } \\
\text { (m) }\end{array}$ & $\begin{array}{l}\text { Thickness } \\
\text { (m) }\end{array}$ \\
\hline \multicolumn{5}{|l|}{ A-1 } \\
\hline A-2 & $6125-6262$ & ITT-GR/ITT-GR & $1831-1873$ & 42 \\
\hline Adda-1 & 7040- 7063 & ITT-GR/ITT-GR & $2112-2119$ & 7 \\
\hline B-1 & absent & ITT-GR/ITT-GR & & \\
\hline E-1 & $6880-7072$ & ITT-GR/ITT-GR & $2060-2118$ & 58 \\
\hline E-2 & no evaluation & & & \\
\hline E-3 & no evaluation & & & \\
\hline G-1 & $6790-6830$ & ITT-GR/ITT-GR & $2032-2045$ & 13 \\
\hline $\mathrm{H}-1$ & 6713- 6981 & ITT-GR/ITT-GR & $2009-2091$ & 82 \\
\hline I-1 & 9182- 9241 & ITT-GR/ITT-GR & $2761-2779$ & 18 \\
\hline M-1 & $6032-6118$ & ITT-GR/ITT-GR & $1805-1831$ & 26 \\
\hline M-2 & no evaluation & & & \\
\hline M-8 & $6152-6256$ & ITT-GR/ITT-GR & $1846-1877$ & 31 \\
\hline $\mathrm{N}-1$ & $6937-7147$ & ITT-GR/ITT-GR & $2082-2146$ & 64 \\
\hline $\mathrm{N}-2$ & no evaluation & & & \\
\hline O-1 & 6254- 7187 & ITT-GR/ITT-GR & $1878-2163$ & 285 \\
\hline P-1 & $9818-9865$ & ITT-GR/ITT-GR & $2955-2969$ & 14 \\
\hline Q-1 & $10378-10730$ & ITT-GR/ITT-GR & $3125-3233$ & 108 \\
\hline Ruth-1 & $5266-5290$ & ITT-GR/ITT-GR & $1570-1577$ & 7 \\
\hline $\mathrm{T}-1$ & 7419- 7519 & ITT-GR/ITT-GR & $2236-2267$ & 31 \\
\hline U-1 & $7430-7594$ & ITT-GR/ITT-GR & $2236-2286$ & 50 \\
\hline V-1 & $7550-7639$ & ITT-GR/ITT-GR & $2268-2295$ & 27 \\
\hline W-1 & 10413-10695 & ITT-GR/ITT-GR & $3140-3226$ & 86 \\
\hline
\end{tabular}

\section{Chalk-6 Unit}

$\begin{array}{ll}\text { Well } & \begin{array}{l}\text { Depth b.KB } \\ \text { (feet) }\end{array} \\ \text { A-1 } & \\ \text { A-2 } & 5950-6125 \\ \text { Adda-1 } & 6866-7040 \\ \text { B-1 } & 7373-7402 \\ \text { E-1 } & 6735-6880 \\ \text { E-2 } & \text { no evaluation } \\ \text { E-3 } & \text { no evaluation } \\ \text { G-1 } & 6610-6790 \\ \text { H-1 } & 6680-6713 \\ \text { I-1 } & 9070-9182 \\ \text { M-1 } & 5902-6032 \\ \text { M-2 } & \text { no evaluation } \\ \text { M-8 } & 6013-6152 \\ \text { N-1 } & 6897-6937 \\ \text { N-2 } & \text { no evaluation } \\ \text { O-1 } & 6097-6254 \\ \text { P-1 } & 9580-9818 \\ \text { Q-1 } & 10058-10378 \\ \text { Ruth-1 } & 5205-5266 \\ \text { T-1 } & 7283-7419 \\ \text { U-1 } & 7300-7434 \\ \text { V-1 } & 7356-7550 \\ \text { W-1 } & 10135-10413 \\ & \end{array}$

Log

(characterizing)

ITT-GR/ITT-GR ITT-GR/ITT-GR ITT-GR/ITT-GR ITT-GR/ITT-GR

ITT-GR/ITT-GR ITT-GR/ITT-GR ITT-GR/ITT-GR ITT-GR/ITT-GR

ITT-GR/ITT-GR ITT-GR/ITT-GR

ITT-GR/ITT-GR ITT-GR/ITT-GR ITT-GR/ITT-GR ITT-GR/ITT-GR ITT-GR/ITT-GR ITT-GR/ITT-GR ITT-GR/ITT-GR ITT-GR/ITT-GR
Depth b.MSL Thickness

(m)

(m)

$\begin{array}{ll}1778-1831 & 52 \\ 2059-2112 & 53 \\ 2212-2220 & 8 \\ 2016-2060 & 44\end{array}$

1978-2032 54

1999-2009 10

2727-2761 34

1765-1805 40

1803-1846 43

2070-2082 12

1830-1878 48

2882-2955 $\quad 73$

3028-3125 97

1551-1570 19

2195-2236 41

2197-2238 41

2208-2268 60

$3055-3140 \quad 85$ 
North Sea Marl

Well
A-1
A-2
Adda-1
B-1
E-1
E-2
E-3
G-1
H-1
I-1
M-1
M-2
M-8
N-1
N-2
O-1
P-1
Q-1
Ruth-1
T-1
U-1
V-1
W-1

Depth b.KB
(feet)

absent

5950- 5952

6841- 6866

absent

6725- 6735

absent

absent

absent

absent

9011- 9070

absent

absent

absent

6839- 6897

absent

6043- 6097

9504- 9580

9971-10072

absent

7228- 7283

7279- 7300

absent

10040-10094
Log

(characterizing)

ITT-GR/GR

ITT-GR/GR

ITT-GR/GR

ITT-GR/GR

ITT-GR/GR

ITT-GR/GR

ITT-GR/GR

ITT-GR/GR

ITT-GR/GR

ITT-GR/GR

ITT-GR/GR
Depth b.MSL Thickness

(m)

(m)

1778-1778

2051-2059 8

2013-2016 3

$2709-2727 \quad 18$

2052-2070 $\quad 18$

1814-1830 16

$2859-2882 \quad 23$

3001-3032 17

2178-2195 $\quad 17$

2190-2197 7

3026-3043 $\quad 17$

\section{CEN-1 Unit}

\begin{tabular}{|c|c|c|c|c|}
\hline Well & $\begin{array}{l}\text { Depth b.KB } \\
\text { (feet) }\end{array}$ & $\begin{array}{l}\text { Log } \\
\text { (characterizing) }\end{array}$ & $\begin{array}{l}\text { Depth b.MSL } \\
\text { (m) }\end{array}$ & $\begin{array}{l}\text { Thickness } \\
\text { (m) }\end{array}$ \\
\hline A-1 & no evaluation & & & \\
\hline A-2 & $5898-5950$ & ITT-GR/ITT-GR & $1762-1778$ & 16 \\
\hline Adda-1 & $6819-6841$ & ITT-GR/ITT-GR & 2044-2051 & 7 \\
\hline B-1 & $7305-7372$ & ITT-GR/ITT-GR & $2191-2211$ & 20 \\
\hline E-1 & $6683-6725$ & ITT-GR/ITT-GR & $2000-2013$ & 13 \\
\hline E-2 & $6530-6547$ & ITT-GR/ITT-GR & $1953-1958$ & 5 \\
\hline E-3 & no evaluation & & & \\
\hline G-1 & $6512-6610$ & ITT-GR/ITT-GR & $1948-1977$ & 29 \\
\hline $\mathrm{H}-1$ & $6670-6680$ & ITT-GR/ITT-GR & 1969-1999 & 3 \\
\hline I-1 & $8942-9011$ & ITT-GR/ITT-GR & 2688-2709 & 21 \\
\hline M-1 & $5832-5910$ & ITT-GR/ITT-GR & $1744-1768$ & 24 \\
\hline M-2 & no evaluation & & & \\
\hline M-8 & no evaluation & & & \\
\hline $\mathrm{N}-1$ & $6800-6839$ & ITT-GR/ITT-GR & $2040-2052$ & 12 \\
\hline $\mathrm{N}-2$ & $6545-6590$ & ITT-GR/ITT-GR & $1964-1977$ & 13 \\
\hline $0-1$ & $6035-6043$ & ITT-GR/ITT-GR & $1811-1814$ & 3 \\
\hline P-1 & $9892-9504$ & ITT-GR/ITT-GR & $2791-2859$ & 68 \\
\hline Q-1 & $9790-9971$ & ITT-GR/ITT-GR & $2946-3001$ & 55 \\
\hline Ruth-1 & $5188-5205$ & ITT-GR/ITT-GR & $1546-1551$ & 5 \\
\hline$T-1$ & $7157-7228$ & ITT-GR/ITT-GR & $2156-2178$ & 22 \\
\hline U-1 & $7233-7279$ & ITT-GR/ITT-GR & $2176-2190$ & 14 \\
\hline$V-1$ & $7300-7356$ & ITT-GR/ITT-GR & 2192-2209 & 17 \\
\hline W-1 & $9945-10040$ & ITT-GR/ITT-GR & $2997-3029$ & 29 \\
\hline
\end{tabular}


CEN-2 Unit

$\begin{array}{lllll}\text { Well } & \begin{array}{l}\text { Depth b.KB } \\ \text { (feet) }\end{array} & \begin{array}{l}\text { Log } \\ \text { (characterizing) }\end{array} & \begin{array}{l}\text { Depth b.MSL } \\ (\mathrm{m})\end{array} & \begin{array}{l}\text { Thickness } \\ (\mathrm{m})\end{array} \\ \text { A-1 } & 5727-5810 & \text { ITT-GR/ITT-GR } & 1736-1761 & 25 \\ \text { A-2 } & 5823-5898 & \text { ITT-GR/ITT-GR } & 1739-1762 & 23 \\ \text { Adda-1 } & 6760-6819 & \text { ITT-GR/ITT-GR } & 2026-2044 & 18 \\ \text { B-1 } & 7230-7305 & \text { ITT-GR/ITT-GR } & 2168-2191 & 23 \\ \text { E-1 } & 6640-6683 & \text { ITT-GR/ITT-GR } & 1987-2000 & 13 \\ \text { E-2 } & 6485-6530 & \text { ITT-GR/ITT-GR } & 1939-1953 & 14 \\ \text { E-3 } & \text { no evaluation } & & & 19 \\ \text { G-1 } & 6450-6512 & \text { ITT-GR/ITT-GR } & 1929-1948 & 19 \\ \text { H-1 } & 6620-6670 & \text { ITT-GR/ITT-GR } & 1981-1996 & 15 \\ \text { I-1 } & 8880-8942 & \text { ITT-GR/ITT-GR } & 2669-2688 & 19 \\ \text { M-1 } & 5787-5832 & \text { ITT-GR/ITT-GR } & 1730-1744 & 14 \\ \text { M-2 } & 5985-6030 & \text { ITT-GR/ITT-GR } & 1793-1807 & 14 \\ \text { M-8 } & 5900-5950 & \text { ITT-GR/ITT-GR } & 1769-1784 & 15 \\ \text { N-1 } & 6765-6800 & \text { ITT-GR/ITT-GR } & 2030-2040 & 10 \\ \text { N-2 } & 6495-6545 & \text { ITT-GR/ITT-GR } & 1948-1964 & 16 \\ \text { O-1 } & 5983-6035 & \text { ITT-GR/ITT-GR } & 1796-1811 & 15 \\ \text { P-1 } & 9200-9282 & \text { ITT-GR/ITT-GR } & 2766-2791 & 25 \\ \text { Q-1 } & 9717-9790 & \text { ITT-GR/ITT-GR } & 2924-2946 & 22 \\ \text { Ruth-1 } & 5132-5188 & \text { ITT-GR/ITT-GR } & 1529-1546 & 17 \\ \text { T-1 } & 7090-7157 & \text { ITT-GR/ITT-GR } & 2136-2156 & 20 \\ \text { U-1 } & 7195-7233 & \text { ITT-GR/ITT-GR } & 2165-2176 & 11 \\ \text { V-1 } & 7256-7300 & \text { ITT-GR/ITT-GR } & 2178-2192 & 14 \\ \text { W-1 } & 9850-9945 & \text { ITT-GR/ITT-GR } & 2968-2997 & 29\end{array}$

\section{CEN-3 Unit}

Well

$\begin{array}{ll}\text { A-1 } & 4800-5727 \\ \text { A-2 } & 4925-5823 \\ \text { Adda-1 } & 5344-6760 \\ \text { B-1 } & 5035-7230 \\ \text { E-1 } & 5460-6640 \\ \text { E-2 } & 5270-6485 \\ \text { E-3 } & \text { no evaluation } \\ \text { G-1 } & 5330-6450 \\ \text { H-1 } & 5255-6620 \\ \text { I-1 } & 5515-8880 \\ \text { M-1 } & 4900-5787 \\ \text { M-2 } & 4980-5985 \\ \text { M-8 } & 4940-5900 \\ \text { N-1 } & 5480-6765 \\ \text { N-2 } & 5340-6495 \\ \text { O-1 } & 4945-5983 \\ \text { P-1 } & 5490-9200 \\ \text { Q-1 } & 6140-9717 \\ \text { Ruth-1 } & 4658-5132 \\ \text { T-1 } & 5397-7090 \\ \text { U-1 } & 5870-7195 \\ \text { V-1 } & 5553-7256 \\ \text { W-1 } & 5600-9850\end{array}$

$\log$

(characterizing)

ITT-GR/ITT-GR

ITT-GR/ITT-GR

ITT-GR/ITT-GR

ITT-GR/ITT-GR

ITT-GR/ITT-GR

ITT-GR/ITT-GR

ITT-GR/ITT-GR ITT-GR/ITT-GR ITT-GR/ITT-GR ITT-GR/ITT-GR ITT-GR/ITT-GR ITT-GR/ITT-GR ITT-GR/ITT-GR ITT-GR/ITT-GR ITT-GR/ITT-GR ITT-GR/ITT-GR ITT-GR/ITT-GR ITT-GR/ITT-GR ITT-GR/ITT-GR ITT-GR/ITT-GR ITT-GR/ITT-GR ITT-GR/ITT-GR
Depth b.MSL Thickness (m) (m)

$\begin{array}{ll}1454-1736 & 282 \\ 1465-1739 & 274 \\ 1595-2026 & 431 \\ 1499-2168 & 669 \\ 1627-1987 & 360 \\ 1569-1939 & 370 \\ & \\ 1587-1929 & 342 \\ 1565-1981 & 416 \\ 1644-2669 & 1025 \\ 1460-1730 & 270 \\ 1487-1793 & 306 \\ 1476-1769 & 293 \\ 1638-2030 & 392 \\ 1596-1948 & 352 \\ 1479-1796 & 317 \\ 1636-2766 & 1130 \\ 1834-2924 & 1090 \\ 1385-1529 & 144 \\ 1620-2136 & 516 \\ 1761-2165 & 404 \\ 1659-2178 & 519 \\ 1673-2968 & 1295\end{array}$




\section{CEN-4 Unit}

$\begin{array}{ll}\text { Well } & \begin{array}{l}\text { Depth b.KB } \\ \text { (feet) }\end{array} \\ \text { A-1 } & 4040-4800 \\ \text { A-2 } & 4134-4925 \\ \text { Adda-1 } & 3893-5344 \\ \text { B-1 } & 4745-5035 \\ \text { E-1 } & 4200-5460 \\ \text { E-2 } & 4210-5270 \\ \text { E-3 } & \text { no evaluation } \\ \text { G-1 } & 3885-5330 \\ \text { H-1 } & 4364-5255 \\ \text { I-1 } & 4928-5515 \\ \text { M-1 } & 4015-4900 \\ \text { M-2 } & 4070-4980 \\ \text { M-8 } & 4028-4940 \\ \text { N-1 } & 4633-5480 \\ \text { N-2 } & 4570-5340 \\ \text { O-1 } & 4087-4945 \\ \text { P-1 } & 4895-5490 \\ \text { Q-1 } & 4997-6140 \\ \text { Ruth-1 } & 3978-4658 \\ \text { T-1 } & 4660-5397 \\ \text { U-1 } & 4870-5870 \\ \text { V-1 } & 3860-5553 \\ \text { W-1 } & 5050-5600\end{array}$

Log

(characterizing)

ITT-GR/ITT-GR ITT-GR/ITT-GR ITT-GR/ITT-GR ITT-GR/ITT-GR ITT-GR/ITT-GR ITT-GR/ITT-GR

ITT-GR/ITT-GR ITT-GR/ITT-GR ITT-GR/ITT-GR ITT-GR/ITT-GR ITT-GR/ITT-GR ITT-GR/ITT-GR ITT-GR/ITT-GR ITT-GR/ITT-GR ITT-GR/ITT-GR ITT-GR/ITT-GR ITT-GR/ITT-GR ITT-GR/ITT-GR ITT-GR/ITT-GR ITT-GR/ITT-GR ITT-GR/ITT-GR ITT-GR/ITT-GR
Depth b.MSL Thickness (m) (m)

$\begin{array}{ll}1222-1454 & 232 \\ 1224-1465 & 241 \\ 1152-1595 & 443 \\ 1411-1499 & 88 \\ 1243-1627 & 384 \\ 1246-1569 & 323 \\ & \\ 1147-1587 & 440 \\ 1293-1565 & 272 \\ 1465-1644 & 179 \\ 1190-1460 & 270 \\ 1209-1487 & 278 \\ 1198-1476 & 278 \\ 1380-1638 & 258 \\ 1362-1596 & 234 \\ 1218-1479 & 261 \\ 1454-1636 & 182 \\ 1485-1834 & 349 \\ 1177-1385 & 208 \\ 1395-1620 & 225 \\ 1456-1761 & 305 \\ 1143-1659 & 516 \\ 1505-1673 & 168\end{array}$

\section{CEN-5 Unit}

$\begin{array}{lllll}\text { Well } & \begin{array}{l}\text { Depth b.KB } \\ \text { (feet) }\end{array} & \begin{array}{l}\text { Log } \\ \text { (characterizing) }\end{array} & \begin{array}{l}\text { Depth b.MSL } \\ \text { (m) }\end{array} & \begin{array}{l}\text { Thickness } \\ \text { (m) }\end{array} \\ \text { A-1 } & 1600-4040 & \text { ITT-GR/ITT-GR } & 478-1222 & 744 \\ \text { A-2 } & 1675-4134 & \text { ITT-GR/ITT-GR } & 475-1224 & 749 \\ \text { Adda-1 } & 1580-3893 & \text { ITT-GR/ITT-GR } & 447-1152 & 705 \\ \text { B-1 } & ? 2430-4745 & \text { ITT-GR/ITT-GR } & ? 705-1411 & 706 \\ \text { E-1 } & 1695-4200 & \text { ITT-GR/ITT-GR } & 479-1243 & 764 \\ \text { E-2 } & 1820-4210 & \text { ITT-GR/ITT-GR } & 518-1246 & 728 \\ \text { E-3 } & \text { no evaluation } & & & \\ \text { G-1 } & 1480-3885 & \text { ITT-GR/ITT-GR } & 414-1147 & 733 \\ \text { H-1 } & 1942-4364 & \text { ITT-GR/ITT-GR } & 555-1293 & 738 \\ \text { I-1 } & 1901-4928 & \text { ITT-GR/ITT-GR } & 542-1465 & 923 \\ \text { M-1 } & 1585-4015 & \text { ITT-GR/ITT-GR } & 450-1190 & 740 \\ \text { M-2 } & 1630-4070 & \text { ITT-GR/ITT-GR } & 466-1209 & 743 \\ \text { M-8 } & 1598-4028 & \text { ITT-GR/ITT-GR } & 458-1198 & 740 \\ \text { N-1 } & 2028-4633 & \text { ITT-GR/ITT-GR } & 586-1380 & 794 \\ \text { N-2 } & 2095-4570 & \text { ITT-GR/ITT-GR } & 607-1362 & 755 \\ \text { O-1 } & 1453-4087 & \text { ITT-GR/ITT-GR } & 415-1218 & 803 \\ \text { P-1 } & 2093-4895 & \text { ITT-GR/ITT-GR } & 600-1454 & 854 \\ \text { Q-1 } & 2075-4997 & \text { ITT-GR/ITT-GR } & 595-1485 & 890 \\ \text { Ruth-1 } & 1750-3978 & \text { ITT-GR/ITT-GR } & 498-1177 & 679 \\ \text { T-1 } & 1720-4660 & \text { ITT-GR/ITT-GR } & 499-1395 & 896 \\ \text { U-1 } & 2073-4870 & \text { ITT-GR/ITT-GR } & 604-1456 & 852 \\ \text { V-1 } & 1340-3860 & \text { ITT-GR/ITT-GR } & 375-1143 & 768 \\ \text { W-1 } & 1944-5050 & \text { ITT-GR/ITT-GR } & 558-1505 & 947\end{array}$


CEN-6 Unit

$\begin{array}{ll}\text { Well } & \begin{array}{l}\text { Depth b.KB } \\ \text { (feet) }\end{array} \\ \text { A-1 } & 177-1600 \\ \text { A-2 } & 263-1675 \\ \text { Adda-1 } & 237-1580 \\ \text { B-1 } & 252-? 2430 \\ \text { E-1 } & 245-1695 \\ \text { E-2 } & 256-1820 \\ \text { E-3 } & \text { no evaluation } \\ \text { G-1 } & 282-1480 \\ \text { H-1 } & 274-1942 \\ \text { I-1 } & 310-1901 \\ \text { M-1 } & 250-1585 \\ \text { M-2 } & 236-1630 \\ \text { M-8 } & 237-1598 \\ \text { N-1 } & 234-2028 \\ \text { N-2 } & 223-2095 \\ \text { O-1 } & 233-1453 \\ \text { P-1 } & 339-2093 \\ \text { Q-1 } & 321-2075 \\ \text { Ruth-1 } & 243-1750 \\ \text { T-1 } & 298-1720 \\ \text { U-1 } & 235-2073 \\ \text { V-1 } & 271-1340 \\ \text { W-1 } & 285-1944 \\ & \end{array}$

Log

(characterizing)

ITT-GR/ITT-GR ITT-GR/ITT-GR ITT-GR/ITT-GR ITT-GR/ITT-GR ITT-GR/ITT-GR ITT-GR/ITT-GR

ITT-GR/ITT-GR ITT-GR/ITT-GR ITT-GR/ITT-GR ITT-GR/ITT-GR ITT-GR/ITT-GR ITT-GR/ITT-GR ITT-GR/ITT-GR ITT-GR/ITT-GR ITT-GR/ITT-GR ITT-GR/ITT-GR ITT-GR/ITT-GR ITT-GR/ITT-GR ITT-GR/ITT-GR ITT-GR/ITT-GR ITT-GR/ITT-GR ITT-GR/ITT-GR
Depth b.MSL Thickness

(m)

(m)

$45-478 \quad 433$

$44-475 \quad 431$

$38-447 \quad 409$

$41-? 705 \quad 664$

$37-479 \quad 442$

$41-518 \quad 477$

$49-414 \quad 365$

$46-555 \quad 509$

$57-542 \quad 485$

$43-450 \quad 407$

$41-466 \quad 425$

$43-458 \quad 415$

$39-586 \quad 547$

$37-607 \quad 570$

$43-415 \quad 372$

$66-600 \quad 534$

$60-595 \quad 535$

$39-498 \quad 459$

$66-499 \quad 433$

$43-604 \quad 561$

$49-375 \quad 326$

$53-558 \quad 555$ 


\subsection{Tables on reservoir parameters}

Recorded and calculated values of the reservoir properties.

\section{Reservoir Parameters for: CA-1 Unit (Early Carboniferous)}

\begin{tabular}{|c|c|c|c|c|c|c|c|c|c|c|c|}
\hline \multirow{3}{*}{$\begin{array}{l}\text { Well } \\
\text { Name }\end{array}$} & \multirow{3}{*}{$\begin{array}{c}\text { Depth } \\
\text { b. KB } \\
\text { FT }\end{array}$} & \multirow{3}{*}{$\begin{array}{l}\text { Depth } \\
\text { b. MSL } \\
\text { M }\end{array}$} & \multirow{3}{*}{$\begin{array}{c}\text { Thickn. } \\
\text { м }\end{array}$} & \multirow{3}{*}{$\begin{array}{l}\text { Unit } \\
\text { no. }\end{array}$} & \multirow{3}{*}{ Shows } & \multirow{3}{*}{$\begin{array}{c}\text { Net.res. } \\
\text { zone } \\
\text { M }\end{array}$} & \multirow{3}{*}{$\begin{array}{l}\text { Log } \\
\text { type }\end{array}$} & \multicolumn{2}{|c|}{ Porosity \% } & \multicolumn{2}{|c|}{ Permeability MD } \\
\hline & & & & & & & & $\log$ & core & & core \\
\hline & & & & & & & & $\emptyset \max \emptyset \min$ & $\bar{\varnothing} \quad \emptyset_{\max } \emptyset_{\min }$ & $K_{\max }$ & Kmin \\
\hline P-1 & $11038-11259$ & $3327-3394$ & 67 & & & 14 & & 155 & 11 & & \\
\hline
\end{tabular}

\section{Reservoir Parameters for: Rotliegendes Group}

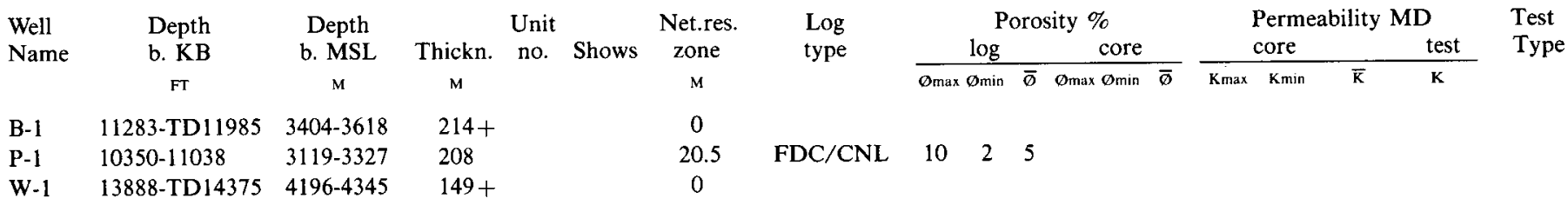

\section{Reservoir parameters for: Zechstein Group}

\begin{tabular}{|c|c|c|c|c|c|c|c|c|c|c|c|c|c|c|c|c|c|}
\hline \multirow{3}{*}{$\begin{array}{l}\text { Well } \\
\text { Name }\end{array}$} & \multirow{3}{*}{$\begin{array}{c}\text { Depth } \\
\text { b. KB } \\
\text { FT }\end{array}$} & \multirow{3}{*}{$\begin{array}{l}\text { Depth } \\
\text { b. MSL } \\
\text { M }\end{array}$} & \multirow{3}{*}{$\begin{array}{c}\text { Thickn. } \\
\text { м }\end{array}$} & \multirow{3}{*}{$\begin{array}{l}\text { Unit } \\
\text { no. }\end{array}$} & \multirow{3}{*}{ Shows } & \multirow{3}{*}{$\begin{array}{c}\text { Net.res. } \\
\text { zone } \\
\text { M }\end{array}$} & \multirow{3}{*}{$\begin{array}{l}\text { Log } \\
\text { type }\end{array}$} & \multicolumn{5}{|c|}{ Porosity \% } & \multicolumn{4}{|c|}{ Permeability MD } & \multirow{3}{*}{$\begin{array}{l}\text { Test } \\
\text { Type }\end{array}$} \\
\hline & & & & & & & & & $\log$ & & core & & & ore & & test & \\
\hline & & & & & & & & $\varnothing \max$ & $\min$ & $\bar{\varnothing}$ & $\emptyset_{\max } \emptyset \min$ & $\bar{\varnothing}$ & $K \max$ & Kmin & $\overline{\mathbf{K}}$ & $\mathbf{K}$ & \\
\hline B-1 & $10190-11283$ & $3009-3404$ & 395 & & & 6 & & 10 & 4 & 5 & & & & & & & \\
\hline Ruth-1 & 5586-TD5618 & $1668-1678$ & $10+$ & & & 0 & & & & & & & & & & & \\
\hline$T-1$ & 7690-TD8713 & $2319-2631$ & $312+$ & & & 0 & & & & & $4 a 0.3 \mathrm{a}$ & $1 \mathbf{a}$ & la & Oa & $\mathrm{Oa}$ & & \\
\hline
\end{tabular}

a) Non res. zone

\section{Reservoir parametres for: Bacton Group}

\begin{tabular}{|c|c|c|c|c|c|c|c|c|c|c|c|c|c|c|c|c|c|}
\hline \multirow{3}{*}{$\begin{array}{l}\text { Well } \\
\text { Name }\end{array}$} & \multirow{3}{*}{$\begin{array}{l}\text { Depth } \\
\text { b. KB } \\
\quad \text { Fr }\end{array}$} & \multirow{3}{*}{$\begin{array}{l}\text { Depth } \\
\text { b. MSL } \\
\text { M }\end{array}$} & \multirow{3}{*}{$\begin{array}{c}\text { Thickn. } \\
\text { M }\end{array}$} & \multirow{3}{*}{$\begin{array}{c}\text { Unit } \\
\text { no. }\end{array}$} & \multirow{3}{*}{ Shows } & \multirow{3}{*}{$\begin{array}{c}\text { Net.res. } \\
\text { zone } \\
\text { м }\end{array}$} & \multirow{3}{*}{$\begin{array}{l}\text { Log } \\
\text { type }\end{array}$} & \multicolumn{5}{|c|}{ Porosity \% } & \multicolumn{4}{|c|}{ Permeability MD } & \multirow{3}{*}{$\begin{array}{l}\text { Test } \\
\text { Type }\end{array}$} \\
\hline & & & & & & & & & $\log$ & & core & & & core & & test & \\
\hline & & & & & & & & $\theta_{\max }$ & $\times \emptyset \min$ & $\bar{\emptyset}$ & $\emptyset_{\max } \emptyset_{\min }$ & $\bar{\varnothing}$ & Kmax & $\mathrm{Kmin}$ & $\overline{\mathrm{K}}$ & $\mathrm{K}$ & \\
\hline B-1 & $9990-10190$ & $3009-3060$ & 51 & & & 0 & & & & & & & & & & & \\
\hline Q-1 & $13910-14620$ & $4203-4420$ & 217 & & + & $\mathbf{a}$ & & & & & & & & & & & \\
\hline $\mathrm{U}-1$ & 14651-TD 16045 & $4437-4862$ & $425+$ & & & $43 b$ & $\mathrm{FDC} / \mathrm{CNL}$ & 11 & $\begin{array}{r}8 \\
14\end{array}$ & 10 & & & & & & & \\
\hline
\end{tabular}

\footnotetext{
a) Very thin. Very poor data

b) Bunter Sandstone Formation

c) Bunter Shale Formation
} 
Reservoir parameters for: J-2 Unit

\begin{tabular}{|c|c|c|c|c|c|c|c|c|c|c|c|c|c|c|c|c|c|}
\hline \multirow{3}{*}{$\begin{array}{l}\text { Well } \\
\text { Name }\end{array}$} & \multirow{3}{*}{$\begin{array}{c}\text { Depth } \\
\text { b. KB } \\
\text { FT }\end{array}$} & \multirow{3}{*}{$\begin{array}{l}\text { Depth } \\
\text { b. MSL } \\
{ }_{M}\end{array}$} & \multirow{3}{*}{$\begin{array}{c}\text { Thickn. } \\
\text { м }\end{array}$} & \multirow{3}{*}{$\begin{array}{l}\text { Unit } \\
\text { no. }\end{array}$} & \multirow{3}{*}{ Shows } & \multirow{3}{*}{$\begin{array}{c}\text { Net.res. } \\
\text { zone } \\
\text { M }\end{array}$} & \multirow{3}{*}{$\begin{array}{l}\text { Log } \\
\text { type }\end{array}$} & \multicolumn{5}{|c|}{ Porosity \% } & \multicolumn{4}{|c|}{ Permeability MD } & \multirow{2}{*}{$\begin{array}{l}\text { Test } \\
\text { Type }\end{array}$} \\
\hline & & & & & & & & & $\log$ & & core & & & core & & test & \\
\hline & & & & & & & & $\emptyset_{\max }$ & $\times \varnothing \min$ & $\overline{\boldsymbol{D}}$ & $\emptyset_{\max } \oslash_{\min }$ & $\bar{\varnothing}$ & $\mathrm{K}_{\max }$ & $\mathrm{Kmin}$ & $\overline{\mathrm{K}}$ & $\mathbf{K}$ & \\
\hline \multicolumn{17}{|c|}{ Upper Member } & \\
\hline A-2 & $9920-9970$ & 2988-3003 & 15 & & & ? & & & & & & & & & & & \\
\hline M-8 & $10143-10260$ & $3062-3098$ & 36 & & & 0 & & & & & & & & & & & \\
\hline $0-1$ & $8901-9425$ & $2685-2845$ & 160 & & & 39 & $\mathrm{FDC} / \mathrm{CNL}$ & 27 & 15 & 25 & & & & & & & \\
\hline U-1 & $10665-10853$ & $3222-3280$ & 58 & & & 0 & & & & & & & & & & & \\
\hline \multicolumn{18}{|c|}{ Lower Member } \\
\hline A-2 & $9970-10050$ & $3003-3027$ & 24 & & + & $?$ & & & & & & & & & & & \\
\hline M-8 & $10260-10467$ & $3098-3161$ & 37 & & + & 3 & $\mathrm{FDC} / \mathrm{CNL}$ & 21 & 18 & 19 & & & & & & $?$ & FT \\
\hline $0-1$ & $9425-9838$ & 2845-2971 & 126 & & & 9 & FDC/CNL & 25 & 18 & 20 & & & & & & & \\
\hline U-1 & $10853-11048$ & $3280-3339$ & 41 & & + & 27 & FDC/CNL & 30 & 15 & 20 & & & & & & 3 & FT \\
\hline
\end{tabular}

Reservoir parameters for: W-1 Unit

\begin{tabular}{|c|c|c|c|c|c|c|c|c|c|c|c|c|c|c|c|c|}
\hline \multirow{3}{*}{$\begin{array}{l}\text { Well } \\
\text { Name }\end{array}$} & \multirow{3}{*}{$\begin{array}{c}\text { Depth } \\
\text { b. KB } \\
\text { Fr }\end{array}$} & \multirow{3}{*}{$\begin{array}{l}\text { Depth } \\
\text { b. MSL } \\
\text { M }\end{array}$} & \multirow{3}{*}{$\begin{array}{c}\text { Thickn. } \\
\text { M }\end{array}$} & \multirow{3}{*}{$\begin{array}{c}\text { Unit } \\
\text { no. }\end{array}$} & \multirow{3}{*}{ Shows } & \multirow{3}{*}{$\begin{array}{c}\text { Net.res. } \\
\text { zone } \\
\text { M }\end{array}$} & \multirow{3}{*}{$\begin{array}{l}\text { Log } \\
\text { type }\end{array}$} & \multicolumn{4}{|c|}{ Porosity \% } & \multicolumn{4}{|c|}{ Permeability MD } & \multirow{3}{*}{$\begin{array}{l}\text { Test } \\
\text { Type }\end{array}$} \\
\hline & & & & & & & & $\log$ & & core & & & ore & & test & \\
\hline & & & & & & & & $\emptyset_{\max } \emptyset_{\min }$ & $\bar{\emptyset}$ & $\emptyset_{\max } \emptyset_{\min }$ & $\bar{\emptyset}$ & $K \max$ & $\overline{K \text { min }}$ & $\overline{\mathbf{k}}$ & $\mathbf{K}$ & \\
\hline W-1 & $13521-13839$ & $4087-4184$ & 97 & & & 67 & FDC & 14 & 11 & & & & & & $?$ & FIT \\
\hline
\end{tabular}

\section{Reservoir parameters for: J-3 Unit}

$\begin{array}{lcrrrrr}\text { Well } & \begin{array}{c}\text { Depth } \\ \text { Name }\end{array} & \begin{array}{c}\text { Depth } \\ \text { b. KB } \\ \text { b. MSL }\end{array} & \begin{array}{c}\text { Thickn. } \\ \text { M }\end{array} & \begin{array}{c}\text { Unit } \\ \text { no. }\end{array} & \text { Shows } & \begin{array}{c}\text { Net.res. } \\ \text { zone } \\ \text { M }\end{array} \\ \text { A-2 } & -9920 & & & & & \text { M } \\ \text { G-1 } & 12037-\text { TD } 12517 & 3632-3778 & 146+ & & + & ? \\ \text { M-8 } & 8940-10143 & 2695-3062 & 367 & & & ? \\ \text { O-1 } & -8901 & -2685 & & & & ? \\ \text { U-1 } & 9595-10665 & 2896-3222 & 326 & & & ? \\ \text { V-1 } & ? 10601-12080 & ? 3198-3648 & ? 450 & & & ?\end{array}$

FDC/CNL $\quad 19$

\section{Reservoir Parameters for: J-4 Unit}

\begin{tabular}{|c|c|c|c|c|c|c|c|c|c|c|c|c|c|c|c|c|}
\hline \multirow[t]{2}{*}{$\begin{array}{l}\text { Well } \\
\text { Name }\end{array}$} & \multirow{2}{*}{$\begin{array}{c}\text { Depth } \\
\text { b. KB } \\
\text { FT }\end{array}$} & \multirow{2}{*}{$\begin{array}{c}\text { Depth } \\
\text { b. MSL } \\
\text { M }\end{array}$} & \multirow{2}{*}{$\begin{array}{c}\text { Thickn. } \\
\text { M }\end{array}$} & \multirow{2}{*}{$\begin{array}{l}\text { Unit } \\
\text { no. }\end{array}$} & \multirow[t]{2}{*}{ Shows } & \multirow{2}{*}{\multicolumn{2}{|c|}{$\begin{array}{c}\text { Net.res. } \\
\text { zone } \\
\text { м }\end{array}$}} & \multirow[t]{2}{*}{$\begin{array}{l}\text { Log } \\
\text { type }\end{array}$} & \multicolumn{3}{|c|}{ Porosity \% } & \multicolumn{4}{|c|}{ Permeability MD } & \multirow[t]{2}{*}{$\begin{array}{l}\text { Test } \\
\text { Type }\end{array}$} \\
\hline & & & & & & & & & $\overline{\theta_{\max }}$ & $\emptyset_{\min }$ & $\overline{\bar{\varnothing}} \emptyset_{\max } \emptyset_{\min } \overline{\bar{c}}$ & $K_{\max }$ & $\mathrm{K} \min$ & $\overline{\mathbf{K}}$ & $\mathrm{K}$ & \\
\hline A-2 & $7470-?$ & & & & + & $?$ & a) & & & & & & & & & \\
\hline Adda-1 & 8400-TD 10005 & $2526-3015$ & $489+$ & & + & 13 & 22 & FDC/CNL & 20 & 14 & 18 & & & & & \\
\hline E-1 & 9727-TD1 3403 & $2928-4048$ & $1120+$ & & + & 38 & 62 & & & & & & & & 0 & DST \\
\hline G-1 & $8088-12037$ & $2429-3632$ & 1204 & & + & 89 & 134 & ITT & 18 & 4 & 11 & & & & & \\
\hline I-1 & 11018-TD12848 & $3321-3879$ & $558+$ & & & 21 & 35 & ITT & 13 & 3 & 7 & & & & & \\
\hline M-1 & 7190-TD7574 & $2158-2275$ & $117+$ & & & $?$ & & & & & & & & & & \\
\hline M-8 & $7517-8940$ & $2262-2695$ & 433 & & & 16 & 26 & & & & & & & & & \\
\hline $0-1$ & $?$ & & & & & $?$ & & & & & & & & & & \\
\hline Q-1 & $?$ & & & & & $?$ & & & & & & & & & & \\
\hline U-1 & 8190- 9595 & $2468-2896$ & 428 & & + & 27 & 44 & FDC/CNL & 19 & 13 & 18 & & & & & \\
\hline $\mathrm{V}-1$ & $9462-10601 ?$ & 2851-3198? & $? 347$ & & & 4 & 7 & & & & & & & & & \\
\hline W-1 & $12333-13521$ & $3725-4087$ & 362 & & + & 7 & 11 & FDC/CNL & 8 & 4 & 5 & & & & & \\
\hline
\end{tabular}


Reservoir parameters for: LC-1 Unit

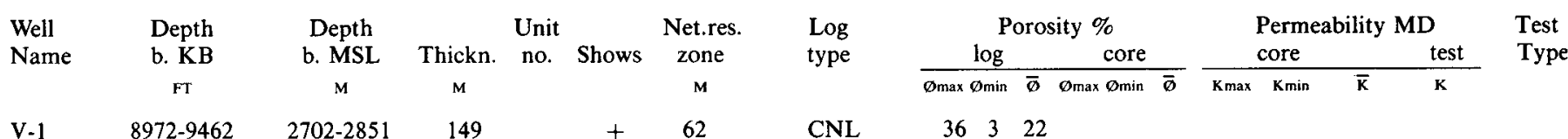

\section{Reservoir parameters for: Valhall Formation}

\begin{tabular}{|c|c|c|c|c|c|c|c|c|c|c|c|c|c|c|c|c|c|c|}
\hline \multirow{3}{*}{$\begin{array}{l}\text { Well } \\
\text { Name }\end{array}$} & \multirow{3}{*}{$\begin{array}{c}\text { Depth } \\
\text { b. KB } \\
\text { FT }\end{array}$} & \multirow{3}{*}{$\begin{array}{l}\text { Depth } \\
\text { b. MSL } \\
\text { M }\end{array}$} & \multicolumn{3}{|c|}{ Unit } & \multirow{3}{*}{$\begin{array}{l}\text { Net.res. } \\
\text { zone } \\
\text { M }\end{array}$} & \multirow{3}{*}{$\begin{array}{l}\text { Log } \\
\text { type }\end{array}$} & \multicolumn{6}{|c|}{ Porosity \% } & \multicolumn{4}{|c|}{ Permeability MD } & \multirow{3}{*}{$\begin{array}{l}\text { Test } \\
\text { Type }\end{array}$} \\
\hline & & & Thickn. & no. & Shows & & & \multicolumn{3}{|c|}{$\log$} & \multicolumn{3}{|c|}{ core } & \multicolumn{3}{|c|}{ core } & \multirow{2}{*}{$\frac{\text { test }}{\mathrm{K}}$} & \\
\hline & & & M & & & & & $\varnothing \max$ & Ømin & $\bar{\varnothing}$ & $\emptyset_{\max }$ & $\theta_{\min }$ & $\vec{\varnothing}$ & $K \max$ & Kmin & $\overline{\mathbf{K}}$ & & \\
\hline A-2 & $7260-7470$ & $2177-2241$ & 64 & & & $?$ & & & & & & & & & & & & \\
\hline Adda-1 & $7530-8400$ & $2262-2526$ & 264 & & + & 22 & FDC/CNL & 36 & 24 & 28 & & & & & & & $\begin{array}{l}8.55 \\
87 a \\
m D f t\end{array}$ & PT \\
\hline E-1 & 8297- 9727 & $2492-2928$ & 436 & & + & 7 & FDC & 24 & 7 & 19 & & & & & & & 0 & $\begin{array}{l}\text { DST } \\
\text { FT }\end{array}$ \\
\hline E-3 & 8350-TD8722 & $2515-2628$ & $113+$ & & & 38 & FDC/CNL & 34 & 19 & 25 & 28 & 17 & 24 & & & & & \\
\hline G-1 & $7410-8088$ & $2222-2429$ & 207 & & & 16 & ITT & & & 17 & & & & & & & & \\
\hline I-1 & $9508-11018$ & $2862-3321$ & 459 & & + & 11 & $?$ & & & 27 & & & & & & & & \\
\hline M-1 & $6902-7190$ & $2071-2159$ & 88 & & & 5 & ITT & 31 & 13 & 26 & & & & & & & & \\
\hline M-7 & 8934-TD9070 & $2691-3733$ & $42+$ & & & 0 & & & & $?$ & & & & & & & & \\
\hline M-8 & $7280-7517$ & $2190-2262$ & 72 & & & 0 & & & & $?$ & & & & & & & & \\
\hline O-1 & $7580-?$ & $2283-?$ & & & & $?$ & & & & & & & & & & & & \\
\hline Q-1 & $?$ & & & & & & & & & & & & & & & & & \\
\hline U-1 & $8112-8190$ & $2445-2468$ & 23 & & + & 7 & FDC/CNL & 30 & 13 & 23 & & & & & & & & \\
\hline V-1 & $8937-8972$ & $2691-2702$ & 12 & & & 9 & $\mathrm{FDC} / \mathrm{CNL}$ & 25 & 9 & 20 & & & & & & & & \\
\hline
\end{tabular}

a) Prod. test 5, test after acid

Reservoir parameters for: Rødby Formation

\begin{tabular}{|c|c|c|c|c|c|c|c|c|c|c|c|c|c|c|c|c|c|}
\hline \multirow{3}{*}{$\begin{array}{l}\text { Well } \\
\text { Name }\end{array}$} & \multirow{3}{*}{$\begin{array}{c}\text { Depth } \\
\text { b. KB } \\
\text { FT }\end{array}$} & \multirow{3}{*}{$\begin{array}{l}\text { Depth } \\
\text { b. MSL } \\
\text { M }\end{array}$} & \multirow{3}{*}{$\begin{array}{c}\text { Thickn. } \\
\text { M }\end{array}$} & \multirow{3}{*}{$\begin{array}{l}\text { Unit } \\
\text { no. }\end{array}$} & \multirow{3}{*}{ Shows } & \multirow{3}{*}{$\begin{array}{c}\text { Net.res. } \\
\text { zone } \\
\text { M }\end{array}$} & \multirow{3}{*}{$\begin{array}{l}\text { Log } \\
\text { type }\end{array}$} & \multicolumn{5}{|c|}{ Porosity \% } & \multicolumn{4}{|c|}{ Permeability MD } & \multirow{3}{*}{$\begin{array}{l}\text { Test } \\
\text { Type }\end{array}$} \\
\hline & & & & & & & & & $\log$ & & core & & & core & & test & \\
\hline & & & & & & & & $\emptyset \max$ & $\varnothing_{\min }$ & $\bar{\emptyset}$ & $\oslash_{\max } \oslash_{\min }$ & $\bar{\varnothing}$ & $\mathrm{K}_{\max }$ & $K_{\min }$ & $\overline{\mathbf{K}}$ & $\mathrm{K}$ & \\
\hline A-2 & $7240-7260$ & $2171-2177$ & 6 & & & $?$ & & & & & & & & & & & \\
\hline Adda-1 & $7512-7530$ & $2256-2262$ & 6 & & & ? & FDC/CNL & 38 & 21 & 29 & & & & & & & \\
\hline E-1 & $8162-8297$ & $2451-2492$ & 41 & & + & $?$ & FDC & 36 & 15 & 23 & & & & & & $\begin{array}{l}\text { none } \\
\text { flow }\end{array}$ & DST \\
\hline E-3 & $8305-8350$ & $2502-2515$ & 13 & & & ? & FDC/CNL & 35 & 20 & 26 & & 18 & & & & & \\
\hline I-1 & $9355-9508$ & $2815-2862$ & 47 & & & $?$ & FDC & 27 & 13 & 20 & & & & & & & \\
\hline M-1 & $6858-6902$ & $2057-2071$ & 14 & & & $?$ & FDC & 21 & 11 & 16 & & & & & & & \\
\hline M-8 & $7212-7280$ & $2169-2190$ & 21 & & & $?$ & FDC/CNL & 40 & 13 & 27 & & & & & & & \\
\hline O-1 & $7570-7580$ & $2280-2283$ & 3 & & & $?$ & ITT & & & & & & & & & & \\
\hline Q-1 & $?$ & & & & & & & & & & & & & & & & \\
\hline $\mathrm{W}-1$ & $?$ & & & & & & & & & & & & & & & & \\
\hline
\end{tabular}


Reservoir parameters for: Chalk Group

\begin{tabular}{|c|c|c|c|c|c|c|c|c|c|c|c|c|c|c|c|c|c|c|}
\hline \multirow{3}{*}{$\begin{array}{l}\text { Well } \\
\text { Name }\end{array}$} & \multirow{3}{*}{$\begin{array}{c}\text { Depth } \\
\text { b. KB } \\
\text { FT }\end{array}$} & \multirow{3}{*}{$\begin{array}{c}\text { Depth } \\
\text { b. MSL } \\
\text { м }\end{array}$} & \multirow{3}{*}{$\begin{array}{l}\text { Thickn. } \\
\text { M }\end{array}$} & \multirow{3}{*}{$\begin{array}{l}\text { Unit } \\
\text { no. St }\end{array}$} & \multirow{3}{*}{ Shows } & \multirow{3}{*}{$\begin{array}{l}\text { Net.res. } \\
\text { zone } \\
\text { M }\end{array}$} & \multirow{3}{*}{$\begin{array}{l}\text { Log } \\
\text { type }\end{array}$} & \multirow{2}{*}{\multicolumn{6}{|c|}{$\begin{array}{l}\text { Porosity } \% \\
\log \quad \text { core }\end{array}$}} & & Perme & eability & $\mathrm{MD}$ & Test \\
\hline & & & & & & & & & & & & & & & core & & test & Type \\
\hline & & & & & & & & $\Phi_{\max }$ & $x \emptyset \min$ & $\bar{\varnothing}$ & $\emptyset_{\max }$ & $\emptyset_{\min }$ & $\bar{\emptyset}$ & Kmax & ax $\mathrm{Kmin}$ & $\overline{\mathrm{K}}$ & $\mathrm{K}$ & \\
\hline A-2 & $5952-6015$ & $1778-1797$ & 19 & $6 \mathrm{U}$ & + & 19 & ITTa & 38 & 27 & 35 & 38 & 12 & 28 & 4 & 0 & 0.1 & $66-166$ & \\
\hline A-2 & $6015-6125$ & $1797-1831$ & 34 & $6 \mathrm{~L}$ & + & 34 & ITTa & 35 & 22 & 31 & 35 & 12 & 22 & 5 & 0 & 0 & $2-28$ & DST \\
\hline A-2 & $6125-6262$ & $1831-1873$ & 42 & 5 & + & 42 & ITTa & 33 & 29 & 30 & & & & & & & 3 & DST \\
\hline A-2 & $6262-6650$ & 1873-1991 & 118 & 4 & + & 118 & ITTa & 30 & 23 & 27 & & & & & & & & \\
\hline Adda-1 & $6866-6959$ & $2059-2087$ & 28 & $6 \mathrm{U}$ & + & 28 & $\mathrm{FDC} / \mathrm{CNL} /$ & & & & & & & & & & & \\
\hline & & & & & & & ITT & 40 & 28 & 37 & & & & & & & & \\
\hline Adda-1 & 6959-7040 & $2087-2112$ & 25 & $6 \mathrm{~L}$ & & 25 & $"$ & 34 & 22 & 28 & & & & & & & & \\
\hline Adda-1 & $7040-7063$ & $2112-2119$ & 7 & 5 & + & 7 & $"$ & 35 & 26 & 29 & & & & & & & 1 & \\
\hline Adda-1 & $7063-7138$ & $2119-2142$ & 23 & 3 & + & 23 & $"$ & 27 & 16 & 22 & & & & & & & & \\
\hline Adda-1 & $7138-7420$ & $2142-2227$ & 85 & 2 & + & 85 & $"$ & 35 & 18 & 24 & & & & & & & 103,51 & \\
\hline Adda-1 & $7240-7512$ & $2227-2556$ & 28 & 1 & + & 28 & $"$ & 28 & 16 & 21 & & & & & & & & \\
\hline E-1 & $6735-6810$ & 2016-2039 & 23 & $6 \mathrm{U}$ & + & 23 & ITT & 36 & 32 & 34 & 39 & 16 & 36 & & 0.2 & 2 & & DST, FT \\
\hline E-1 & $6810-6880$ & $2039-2060$ & 21 & $6 \mathrm{~L}$ & + & 21 & ITT & 32 & 22 & 29 & 37 & 12 & 30 & & 20.03 & 1 & & DST, FT \\
\hline E-1 & $6880-7072$ & $2060-2118$ & 58 & 5 & + & 58 & ITT & 37 & 27 & 31 & 33 & 25 & 30 & 5 & $5 \quad 2$ & 3 & & DST \\
\hline E-1 & $7072-7325$ & $2118-2195$ & 77 & 4 & + & 77 & ITT & 31 & 21 & 27 & & & & & & & & \\
\hline E-1 & $7325-7500$ & $2195-2249$ & 54 & 3 & + & 54 & ITT & 24 & 16 & 20 & & & & & & & & \\
\hline E-1 & $7500-8115$ & $2249-2436$ & 187 & 2 & + & 187 & ITT & 28 & 17 & 22 & & & & & & & & \\
\hline E1 & $8115-8162$ & $2436-2451$ & 14 & 1 & + & 14 & ITT & 18 & 16 & 18 & & & & & & & & DST \\
\hline G-1 & $6610-6675$ & $1978-1997$ & 19 & $6 \mathrm{U}$ & + & 19 & ITT/GR & 37 & 33 & 36 & 38 & 25 & 36 & & 0.13 & 1 & 0.45 & DST \\
\hline G-1 & $6675-6790$ & $1997-2032$ & 35 & $6 \mathrm{~L}$ & + & 35 & ITT/GR & 34 & 20 & 29 & 39 & 20 & 30 & & 0.03 & 0.3 & 0.07 & DST \\
\hline G-1 & $6790-6830$ & $2032-2045$ & 13 & 5 & + & 13 & ITT/GR & 32 & 23 & 29 & & & & & & & & DST \\
\hline G-1 & $6830-6875$ & $2045-2058$ & 13 & 4 & + & 13 & ITT/GR & 25 & 20 & 23 & & & & & & & & \\
\hline G-1 & $6875-7120$ & $2058-2133$ & 75 & 3 & + & 75 & ITT/GR & 22 & 19 & 21 & & & & & & & & \\
\hline G-1 & $7120 \cdot 7410$ & $2133-2222$ & 89 & 2 & + & 89 & ITT/GR & 27 & 23 & 25 & & & & & & & & \\
\hline $\mathrm{H}-1$ & $6680-6713$ & 1999-2009 & 10 & $6 \mathrm{~b}$ & + & 10 & FDC & 49 & 42 & 46 & 46 & 40 & 43 & 12 & 1 & 3 & & DST \\
\hline H-1 & $6713-6981$ & $2009-2091$ & 82 & 5 & + & 82 & FDC & 49 & 22 & 36 & 46 & 32 & 39 & 17 & 3 & 7 & & DST \\
\hline H-1 & 6981-TD7100 & $2091-2127$ & $36+$ & 4 & + & 36 & FDC & 29 & 18 & 24 & & & & & & & & \\
\hline I-1 & $9070-9138$ & $2727-2748$ & 21 & $6 \mathrm{U}$ & + & 21 & ITT & 33 & 26 & 30 & 32 & 15 & 29 & & 0.05 & 0.3 & & DST \\
\hline I-1 & $9138-9182$ & $2748-2761$ & 13 & $6 \mathrm{~L}$ & + & 13 & ITT & 28 & 9 & 24 & 28 & 5 & 17 & 0.23 & 0.01 & 0.05 & & DST \\
\hline I-1 & $9182-9241$ & $2761-2779$ & 18 & 5 & + & 18 & ITT & 32 & 21 & 28 & 24 & 18 & 20 & & 0.01 & 0.1 & 30 & DST \\
\hline I-1 & $9241-9355$ & $2779-2814$ & 35 & 4 & + & 35 & ITT & 28 & 18 & 25 & & & & & & & & \\
\hline M-1 & $5902-5962$ & $1765-1784$ & 19 & $6 \mathrm{U}$ & + & 19 & ITT & 42 & 36 & 39 & 42 & 23 & 35 & & 0.02 & 2 & & \\
\hline M-1 & $5962 \cdot 6032$ & $1784-1805$ & 21 & $6 \mathrm{~L}$ & + & 21 & ITT & 42 & 31 & 34 & 37 & 17 & 27 & & 0.01 & 0.2 & & \\
\hline M-1 & $6032-6118$ & $1805-1831$ & 26 & 5 & + & 26 & ITT & 42 & 35 & 40 & 45 & 25 & 35 & 10 & 2 & 5 & & \\
\hline M-1 & $6118-6471$ & $1831-1939$ & 108 & 4 & + & 108 & ITT & 34 & 31 & 32 & 33 & 20 & 27 & 9 & 0.3 & 2 & & FT \\
\hline M-1 & $6471-6590$ & $1939-1975$ & 36 & 3 & + & 36 & ITT & 33 & 31 & 31 & 27 & 21 & 24 & 4 & $\quad 1$ & 2 & & FT \\
\hline M-1 & $6590-6858$ & $1975-2057$ & 82 & 2 & & 82 & ITT & 31 & 29 & 30 & & & & & & & & \\
\hline $\mathrm{N}-1$ & 6897- 6918 & $2070-2076$ & 6 & $6 \mathrm{U}$ & + & 6 & ITT/GR & 35 & 31 & 34 & 37 & 18 & 28 & & 0.16 & 3 & & FT \\
\hline$N-1$ & $6918-6937$ & 2076-2082 & 6 & $6 \mathrm{~L}$ & + & 6 & ITT/GR & 33 & 25 & 30 & $?$ & $?$ & 27 & 0.35 & 0.18 & 0.26 & & FT \\
\hline N-1 & 6937- 7147 & $2082-2146$ & 64 & 5 & + & 64 & ITT/GR & 37 & 32 & 26 & 34 & 33 & 34 & 4 & 3 & 3 & & FT \\
\hline N-1 & $7147-7745$ & $2146-2328$ & 182 & 4 & + & 182 & ITT/GR & 32 & 25 & 28 & & & & & & & & \\
\hline $\mathrm{N}-1$ & 7745- 7904 & $2328-2377$ & 49 & 3 & & 49 & ITT/GR & 24 & 22 & 23 & & & & & & & & \\
\hline N-1 & 7904-TD8155 & $2377-2453$ & $76+$ & 2 & & 76 & ITT/GR & 24 & 22 & 23 & & & & & & & & \\
\hline O-1 & $6097-6180$ & $1830-1856$ & 26 & $6 \mathrm{U}$ & & 26 & $\mathrm{CNL}$ & & & 27 & 32 & 19 & 25 & & 0.01 & 0.05 & & \\
\hline $0-1$ & $6180-6254$ & $1856-1878$ & 22 & $6 \mathrm{~L}$ & & 22 & $\mathrm{CNL}$ & & & 25 & & & & & & & & \\
\hline $0-1$ & $6254-7187$ & $1878-2163$ & 285 & 5 & & 285 & $\mathrm{CNL}$ & 28 & 25 & 26 & & & & & & & & \\
\hline $0-1$ & 7187- 7349 & $2163-2212$ & 49 & 4 & & 49 & $\mathrm{CNL}$ & 23 & 21 & 22 & & & & & & & & \\
\hline $\mathrm{O}-1$ & $7349-7408$ & $2212-2230$ & 18 & 3 & & 18 & $\mathrm{CNL}$ & 22 & 21 & 21 & & & & & & & & \\
\hline $0-1$ & $7408-7500$ & $2230-2258$ & 28 & 2 & & 28 & $\mathrm{CNL}$ & & & 21 & & & & & & & & \\
\hline O-1 & $7500-7580$ & $2258-2282$ & 24 & 1 & & 24 & $\mathrm{CNL}$ & & & 21 & & & & & & & & \\
\hline$P-1$ & $9580-9745$ & $2882-2932$ & 50 & $6 \mathrm{U}$ & + & 50 & ITT & 39 & 33 & 37 & & & & & & & & \\
\hline$P-1$ & 9745- 9818 & $2932-2955$ & 23 & $6 \mathrm{~L}$ & + & 23 & ITT & 35 & 33 & 33 & & & & & & & & \\
\hline$P-1$ & $9818-9865$ & $2955-2969$ & 14 & 5 & + & 14 & ITT & & & 35 & & & & & & & & \\
\hline $\mathrm{P}-1$ & $9865-10350$ & $2969-3117$ & 148 & 4 & + & 148 & ITT & 31 & 27 & 30 & & & & & & & & \\
\hline Q-1 & 10058-10195 & $3028-3097$ & 69 & $6 \mathrm{U}$ & + & 69 & ITT & 25 & 23 & 24 & 17 & 3 & 13 & 0.29 & 0.01 & 1 & & \\
\hline Q-1 & $10195-10378$ & $3097-3125$ & 28 & $6 \mathrm{~L}$ & + & 28 & ITT & 20 & 16 & 18 & & & & & & & & \\
\hline Q-1 & $10378-10730$ & $3125-3233$ & 108 & 5 & & 108 & ITT & 20 & 16 & 19 & & & & & & & & \\
\hline Q-1 & $10730-11640$ & $3233-3510$ & 277 & 4 & & 277 & ITT & 19 & 12 & 14 & & & & & & & & \\
\hline Q-1 & $11640-12128$ & $3510-3659$ & 149 & 3 & & 149 & ITT & 13 & 11 & 12 & & & & & & & & \\
\hline
\end{tabular}




$\begin{array}{lrrrlrr}\text { Q-1 } & 10378-10730 & 3125-3233 & 108 & 5 & & 108 \\ \text { Q-1 } & 10730-11640 & 3233-3510 & 277 & 4 & & 277 \\ \text { Q-1 } & 11640-12128 & 3510-3659 & 149 & 3 & & 149 \\ \text { Q-1 } & 12128-13000 & 3659-3925 & 265 & 2 & & 265 \\ \text { T-1 } & 7283-7355 & 2195-2217 & 22 & 6 \mathrm{U} & + & 22 \\ \text { T-1 } & 7355-7419 & 2217-2236 & 19 & 6 \mathrm{~L} & + & 19 \\ \text { T-1 } & 7419-7519 & 2236-2267 & 31 & 5 & + & 31 \\ \text { T-1 } & 7519-7685 & 2267-2317 & 50 & 4 & + & 50 \\ \text { U-1 } & 7300-7360 & 2197-2215 & 18 & 6 \mathrm{U} & + & 18 \\ \text { U-1 } & 7360-7434 & 2215-2238 & 23 & 6 \mathrm{~L} & + & 23 \\ \text { U-1 } & 7434-7594 & 2238-2286 & 48 & 5 & + & 48 \\ \text { U-1 } & 7594-7800 & 2286-2349 & 63 & 4 & + & 63 \\ \text { U-1 } & 7800-7898 & 2349-2379 & 30 & 3 & + & 30 \\ \text { U-1 } & 7898-8109 & 2379-2444 & 65 & 2 & + & 65 \\ \text { V-1 } & 7355-7470 & 2208-2243 & 39 & 6 \mathrm{U} & + & 35 \\ \text { V-1 } & 7470-7550 & 2243-2268 & 35 & 6 \mathrm{~L} & + & 35 \\ \text { V-1 } & 7550-7639 & 2268-2295 & 27 & 5 & + & 27 \\ \text { V-1 } & 7639-8025 & 2295-2412 & 117 & 4 & + & 117 \\ \text { V-1 } & 8025-8375 & 2412-2519 & 107 & 3 & + & 107 \\ \text { V-1 } & 8375-8937 & 2519-2690 & 171 & 2 & + & 171 \\ \text { W-1 } & 10135-10249 & 3055-3090 & 35 & 6 \mathrm{U} & + & 35 \\ \text { W-1 } & 10249-10413 & 3090-3140 & 50 & 6 \mathrm{~L} & + & 50 \\ \text { W-1 } & 10413-10695 & 3140-3226 & 86 & 5 & + & 86 \\ \text { W-1 } & 10695-11267 & 3226-3400 & 174 & 4 & + & 174 \\ \text { W-1 } & 11267-11782 & 3400-3557 & 157 & 3 & + & 157 \\ \text { W-1 } & 11782-12333 & 3557-3725 & 168 & 2 & + & 168\end{array}$

$\begin{array}{llll}\text { ITT } & 20 & 16 & 19\end{array}$

$\begin{array}{llll}\text { ITT } & 19 & 12 & 14\end{array}$

$\begin{array}{llll}\text { ITT } & 13 & 11 & 12\end{array}$

$\begin{array}{llll}16 & 13 & 15\end{array}$

$\begin{array}{llllllllll}\text { FDC/CNL } & 30 & 19 & 23 & 32 & 12 & 27 & 8 & 0.007 & 0.2\end{array}$

$\begin{array}{lllllllllll}\text { ITT } & 26 & 18 & 21 & 38 & 8 & 21 & 10 & 0.006 & 0.07\end{array}$

$\begin{array}{llllllllll}\text { ITT } & 28 & 14 & 20 & 31 & 12 & 23 & 20 & 0.007\end{array}$

$\begin{array}{lllllllllll}\text { ITT } & 27 & 14 & 18 & 28 & 2 & 18 & 19 & 0.005 & 2 & 5\end{array}$

FDC/CNL $30 \quad 23 \quad 26$

FDC/CNL $27 \quad 21 \quad 25$

FDC/CNL $25 \quad 24 \quad 24$

FDC/CNL $24 \quad 20 \quad 22$

FDC/CNL $24 \quad 20 \quad 22$

FDC/CNL $26 \quad 17 \quad 21$

FDC/CNL $25 \quad 23 \quad 25$

FDC/CNL $25 \quad 23 \quad 24$

FDC/CNL $24 \quad 22 \quad 23$

FDC/CNL $24 \quad 22 \quad 23$

FDC/CNL $24 \quad 20 \quad 22$

FDC/CNL $24 \quad 8 \quad 14$

$\begin{array}{llll}\text { FDC/CNL } & 13 & 12 & 12\end{array}$

$\begin{array}{llll}\text { FDC/CNL } & 13 & 12 & 13\end{array}$

FDC/CNL $17 \quad 11 \quad 13$

FDC/CNL $10 \quad 6 \quad 8$

FDC/CNL $84 \quad 4 \quad 5$

FDC/CNL $12 \quad 6 \quad 9$

a) No logs below $6650^{\circ}$. Base chalk at $7230^{\prime}$ b. $\mathrm{KB}$

b) Unit 6 cannot be subdivided 


\subsection{References}

Al-Kasim, F., Rønnevik, H.C., and Ulleberg, K. 1975: Review of the Jurassic Offshore Norway. - NPF-Jurassic Northern North Sea Symposium, Stavanger.

Andeev, P.F., Bogomolov, A.I., Dobryanskii, A.F., and Kartsev, A.A. 1968: Transformation of petroleum in nature. - Pergamon Press.

Barnard, P.C. and Cooper, B.S. 1981: Oils and Source Rocks of the North Sea area. - In: Illing, L.V. \& Hobson, G.D. (eds.): Petroleum Geology of the Continental shelf of North-West Europe. - Heyden \& Son Ltd. for Inst. of Petroleum, London, pp. 169-175.

Bertelsen, F. 1975: Triassic palynology and stratigraphy of some Danish North Sea boreholes. - Danm. geol. Unders., Årbog 1974, pp. 17-32.

Bertelsen, F. 1978: Carboniferous miospores from the Nordsø P-1 borehole, Danish North Sea offshore area. - Danm. geol. Unders., Årbog 1977, pp. 101-111.

Bertelsen, F. 1980: Lithostratigrpahy and depositional history of the Danish Triassic. - Danm. geol. Unders., Ser. B, 4, 59 p.

Blair, D.G. 1975: Structural styles in North Sea oil and gas fields. In: Woodland, A.W. (ed.): Petroleum and the Continental Shelf of NW Europe, vol. 1, Geology - Applied Sci., pp. 327-335.

Bowen, J.M. 1975: The Brent Oil-Field. - In: Woodland, A.W. (ed.): Petroleum and the Continental shelf of NW Europe, vol. 1, Geology - Applied Sci., pp. 353-360.

Brennand, T.P. \& Veen, R.F. 1975: The Ank Oil-Field. - In: Woodland, A.W. (ed.): Petroleum and the Continental Shelf of NW Europe, vol. 1, Geology - Applied Sci., pp. 275-281.

Brooks, J.R.V. \& Chesher, J.A. 1975: Review of the Offshore Jurassic of the UK Northern North Sea. - NPF-Jurassic Northern North Sea Symposium, Stavanger.

Childs, F.B. \& Reed, P.E.C. 1975: Geology of the Dan Field and the Danish North Sea. Danm. geol. Unders., III. række, 43, 24 p.

Cottencon, A., Parant, B. \& Flaceliere, B. 1975: Lower Cretaceous gas fields in Holland. - In: Woodland, A.W. (ed): Petroleum and the Continental Shalf of NW Europe, vol. 1, Geology - Applied Sci., pp. 403-412.

Day, G.A., Cooper, B.A., Andersen, C., Burgers, W.F.J., Rønnevik, H.G. \& Schønich, H. 1981: Regional Seismic Structure Maps of the North Sea. - In: Illing, L.V. \& Hobson, G.D. (eds.): Petroleum Geology of the Continental shelf of North-West Europe. - Heyden \& Son Ltd. for Inst. of Petroleum, London, pp. 76-84.

Deegan, C.E. \& Scull, B.J. (compilers) 1977: A proposed standard lithostratigraphic nomenclature for the Central and Northern North Sea. - Rep. Inst. Geol. Sci., No. 77/25.

Dickinson, W.R. 1974: Plate tectonics and sedimentation. - In: Tectonics and sedimentation: SEPM Spec. Pub. 22, pp. 1-27.

Dixon, J.E., Fitton, J.G. \& Frost, R.T.C. 1981: The tectonic significance of post-Carboniferous igneous activity in the North Sea Basin. - In: Illing, L.V. \& Hubson, G.C. (eds.): Petroleum Geology of the Continental shelf of North-West Europe. Heyden \& Son (Ltd. for Inst. of Petroleum, London, pp. 121137.

Donato, J.A. \& Tilly, M.C. 1981: A Regional Interpretation of North Sea Gravity data. - In: Illing, L.V. \& Hobson, G.D. (eds.): Petroleum Geology of the Continental shelf of NorthWest Europe. - Heyden \& Son Ltd. for Inst. of Petroleum, London, pp. 65-75.

Dow, W.G. 1977: Kerogen studies and geological interpretations. - J. geochem. Explor., vol. 7, pp. 79-99.
Eames, T.D. 1975: Coal Rank and gas source relationshipsRotliegendes reservoirs. - In: Woodland, A.W. (ed.): Petroleum and the Continental Shelf of NW Europe, vol. 1, Geology Applied Sci., pp. 191-203.

Evans, T.R. \& Coleman, N.C. 1974: North Sea Geothermal Gradients. - Nature, vol. 247, pp. 28-230.

Eynon, G. 1981: Basin Development and Sedimentation in the Middle Jurassic of the Northern North Sea. - In: Illing, L.V. \& Hobson, G.D. (eds.): Petroleum Geology of the Continental shelf of North-West Europe. - Heyden \& Son Ltd. for Inst. of Petroleum, London, pp. 196-204.

Faerseth, R.B., McIntyre, R.M. \& Naterstad, J. 1976: Mesozoic alkaline dykes in the Sunnhærdland region western Norway: ages, geochemistry and regional significance. - Lithos, 9, pp. 331-348.

Frost, R.t.C., Fritch, F.J. \& Miller, J.A. 1981: The age and nature of the crystalline basement of the North Sea Basin. - In: Illing, L.V. \& Hobson, G.D. (eds.): Petroleum Geology of the Continental shelf of North-West Europe. - Heyden \& Son Ltd. for Inst. of Petroleum, London, pp. 43-57.

Fuller, J.G.C.M. 1975: Jurassic source rock potential and hydrocarbon correlations, North Sea. - In: Finstad, K.G. \& Selley, R.C. (eds.): Jurassic Northern North Sea Symposium, Stavanger. Proceedings of the Norwegian Petroleum Society, Paper No. 11, 18 p.

Fyfe, J.A., Abbots, I. \& Crosby, A. 1981: The Subcrop of the MidMesozoic Unconformity in the UK Area. - In: Illing, L.V. \& Hubson, G.D. (eds.): Petroleum Geology of the Continental shelf of North-West Europe. - Heyden \& Son Ltd. for Inst. of Petroleum, London, pp. 236-244.

Gaida, K.-H., Kemper, E. \& Zimmerle, W. 1978: Das Oberapt von Sarstedt und seine Tuffe. - Geol. Jahrb., A 45, pp. 43-123.

Hancock, N.J. \& Taylor, A.M. 1978: Clay mineral diagenesis and oil migration in the Middle Jurassic Brent Sand Formation. - J1. Geol. Soc. London, 135, pp. 69-72.

Harding, T.P. \& Lowell, J.D. 1979: Structural styles, their platetectonic habitats, and hydrocarbon traps in petroleum provinces. - AAPG Bull., vol. 63, No. 7, pp. 1016-1058.

Heritier, F.E., Lossel, P. \& Wathne, E. 1981: The Frigg Gas-Field. - In: Illing, L.V. \& Hobson, G.D. (eds.): Petroleum Geology of the Continental shelf of North-West Europe. - Heyden \& Son Ltd. for Inst. of Petroleum, London, pp. 380-391.

Harms, J.C., Tackenberg, P., Pickles, E. \& Pollock, R.E. 1981: The Brae Oilfield Area. - In: Illing, L.V. \& Hobson, G.D. (eds.): Petroelum Geology of the Continental shelf of North-West Europe. - Heyden \& Son Ltd. for Inst. of Petroleum, London, pp. 352-357.

Hatch, L.F. and Mater, S. 1977: From hydrocarbons to petrochemicals. Part 3: Refinery processes and petrochemicals. Hydrocarbon processing No. 56, pp. 191-201.

Hood, A., Gutjahr, C.C.M., Heacock, R.L. 1975: Organic Metamorphism and the Generation of Petroleum. - AAPG Bull. vol. 59, No. 6, pp. 986-996.

Larsen, G. 1966: Rhaetic-Jurassic-Lower Cretacreous Sediments in the Danish Embayment (A Heavy-Mineral Study). - Danm. geol. Unders., II. række, 91, 127 p.

Larsen, O. 1972: Kalium/Argon datering af prøver fra danske dybdeboringer. - Dansk geol. Foren. Årsskrift for 1971, pp. 9194.

Madsen, L. 1975: Approximate geothermal gradients in Denmark and the Danish North Sea Sector. - Danm. geol. Unders., Årbog 1974, pp. 5-16. 
Marie, J.P.P. 1976: Rotliegendes stratigraphy and diagenesis. - In: Illing, L.V. \& Hobson, G.D. (eds.): Petroleum Geology of the Continental shelf of North-West Europe. - Heyden \& Son Ltd. for Inst. of Petroleum, London, pp. 205-210.

Michelsen, O. 1975: Lower Jurassic biostratigraphy and ostracods of the Danish Embayment. - Danm. geol. Unders., II. række, $104,287 \mathrm{p}$.

Michelsen, O. 1976: Kortfattet oversigt over de geologiske forhold i den danske del af Nordsøen. - Danm. geol. Unders., Årbog 1975, pp. 117-132.

Michelsen, O. 1978a: The Lower Jurassic of the Dansk Nordsø O1 boring, Central Trough. - Danm. geol. Unders., Årbog 1977, pp. 77-87.

Michelsen, O. 1978b: Stratigraphy and distribution of Jurassic deposits of the Norwegian-Danish Basin. - Danm. geol. Unders., Ser.B, 2, 28 p.

Millot, G. 1970: Geology of clays. - Springer Verlag, London, 429 p.

Oftedahl, C. 1978: Origin of the magmas of the Vestfold lava plateau. - In: Neumann, E.-R. \& Ramberg I.B. (eds.): Petrology and Geochemistry of the Continental Rifts. - D. Riedel Publishing Co., Dordrecht, Holland, pp. 193-208.

Olaussen, S. 1981: Marine incursion in Upper Palaeozoic sedimentary rocks of the Oslo Region, Southern Norway. - Geol. Mag. 118 (3), pp. 281-288.

Pennington, J.J. 1975: The Geology of the Argyll Field. - In: Woodland, A.W. (ed.): Petroleum and the Continental Shelf of North-West Europe, vol. 1, Geology - Applied Sci., pp. 285-291.

Oudin, J. 1976: Geochemical study of the North Sea Basin. - Bull. Centre Rech. Pau-SNPA, 10, pp. 339-358.

Rasmussen, L.B. 1974: Some geological results from the first five Danish exploration wells in the North Sea. - Danm. geol. Unders., III. række, 42, $46 \mathrm{p}$.

Rasmussen, L.B. 1978: Geological aspects of the Danish North Sea Sector. - Danm. geol. Unders. III. række, 44, 85 p.

Rhys, G.H. 1974: A proposed standard lithostratigraphic nomenclature for the Southern North Sea and an outline structural nomenclature for the whole of the (UK) North Sea. - Inst. Geol. Sci., Report 74/8, 114 p.

Richter-Bernburg, G. 1955: Stratigraphische Gliederung des deutschen Zechsteins. - Z. deutsch. geol. Ges. Band 15, 4, pp. 843854.

Sarkisyan, S.G. 1970: Origin of authigenic clay minerals and their significance in petroleum geology. - Sedimentary Geology 7 , pp. 1-22.

Scholle, P.A. 1977a: Deposition, diagenesis, and hydrocarbon potential of Ädeep-water $\ddot{A}$ limestones. - AAPG, Continuing Education Course, Series No. 7, 25 p
Scholle, P.A. 1977b: Current oil and gas production from North American Upper Cretaceous Chalks. - US Department of the Interior. Geol. Surv. Circular 767, 51 p.

Skarpnes, O., Hamar, G.P., Jakobsson, K.H. \& Ormaasen, D.E. 1980: Regional Jurassic Setting of the North Sea North of the Central Highs. - NPF Sedimentation of the North Sea Reservoir Rocks. Symposium Geilo, $7 \mathrm{p}$.

Stach, E., Mackowsky, M.-Th., Teichmuller, M., Taylor, G.H., Chandra, D., Teichmuller, R. 1975: Strach's Textbook of Coal Petrology. - Gebruder Borntraeger. Berlin. Stuttgart.

Taylor, J.C.M. 1981: Zechstein facies and petroleum ptrospects in the Central and Northern North Sea. - In: Illing, L.V. \& Hobson, G.D. (eds.): Petroleum Geology of the Continental shelf of North-West Europe. - Heyden \& Son Ltd. for Inst. of Petroeum, London, pp. 176-181.

Tissot, B. \& Welte, D.H. 1978: Petroleum formation and occurrence. A new approach to oil and gas exploration. - Springer Verlag. New York, $538 \mathrm{p}$

Weaver, C.F. 1960: Possible uses of clay minerals in search for oil. - Bull. Amer Assoc. petrol. Geol. 44, pp. 1505-1518.

Weismann, T.J. 1979: Developments in Geochemistry and their contributions to hydrocarbon exploratoin. - 10th World Pet. Congr. Paper 2, Bucharest, 18 p.

Werner, A.G. 1786: Allgemeine Betrachtungen uber die festen Erdkorper. - Schriften der mineralogischen Gesellschaft in Dresden. Band 1.

Whiteman, A.J., Rees, G., Naylor, D. \& Pegrum, R.M. 1975 North Sea troughs and plate tectonics. - Norges geol. Unders., 316 , pp. 137-161.

Woodhall, D. \& Knox, R.W.O'B. 1979: Mesozoic volcanism in the northern North Sea and adjacent areas. - Bull. Geol. Surv. GB, 70, pp. 34-56.

Ziegler, P.A. 1975: North Sea Basin History in the Tectonic Framework of North-Western Europe. - In: Woodland, A.W. (ed.): Petroleum and the Continental Shelf of North-West Europe, vol. 1, Geology - Applied Sci., pp. 131-148.

Ziegler, P.A. 1977: Geology and hydrocarbon provinces of the North Sea. - Geo Journal, 1, pp. 7-32.

Ziegler, P.A. 1978: North-Western Europe: Tectonics and basin development. - In: van Loon, A.J. (ed.): Key-notes of the MEGS-II Geol. Mijnbouw, 57, pp. 589-626.

Ziegler, P.A. 1981: Evolution of sedimentary basins in North-West Europe. - In: Illing, L.V. \& Hobson, G.D. (eds.): Petroleum Geology of the Continental shelf of North-West Europe. Heyden \& Son Ltd. for Inst. of Petroleum, London, pp. 3-39. 


\section{List of figures}

Fig. 1 Location of wells mentioned in the present publication, and the status of the wells by October 1st, $1981 \ldots \ldots$.

Fig. 2 Standard stratigraphic subdivison of formations in the Danish Central Graben with indications of oil and gas shows ...................

Fig. 3 Legend of symbols used for a) lithology, b) structures, and c) wells ....

Fig. 4 Structural outline of the Danish area (from Michelsen 1978b) ..........

Fig. 5 a) Structural outline of the Danish Central Graben at the base Zechstein level and b) Bouguer gravity map of the Graben area (after Edcon) ........ . 10-11

Fig. 6 Two-way time map for Top Chalk ..

Fig. 7 a) Location map and legend of geosections presented in figs. 7 to 13 and b) Geosection, line $5423 \ldots \ldots \ldots \ldots$.

Fig. 8 Geosections a) line 0503 and b) line $5260 \ldots \ldots \ldots \ldots \ldots \ldots \ldots$

Fig. 9 Geosections a) line 5220 and b) line 75 DK-40 ..................

Fig. 10 Line 0453 a) seismic section b) geosec-

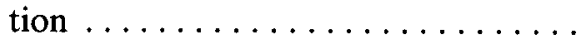

Fig. 11 Line 75-DK-45 a) seismic section b)

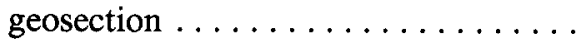

Fig. 12 Line 0508S a) seismic section b) geosection ...................

Fig. 13 Line 5310 a) seismic section b) geosection ....................

Fig. 14 Generalized isopach map of the Jurassic sequence .............

Fig. 15 Generalized isopach map of the Lower Cretaceous sequence ...........

Fig. 16 Generalized isopach map of the Chalk Group ..................

Fig. 17 Palinspastic profile of Pre-Upper-Permian deposits ...............

Fig. 18 Lithological profiles of the Rotliegendes deposits ............

Fig. 19 Palinspastic profile of the Zechstein deposits $\ldots \ldots \ldots \ldots \ldots \ldots \ldots$

Fig. 20 Palinspastic profile of the Triassic de-

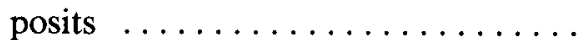

Fig. 21 Distribution and thickness of a) the Fjerritslev Formation and b) the J-2 Unit $\ldots \ldots \ldots \ldots \ldots \ldots \ldots$

Fig. 22 Distribution and thickness of the a) J-3 Unit and b) the J-4 Unit .........

Fig. 23 Palinspastic profile of Jurassic deposits
Fig. 24 Palinspastic profile of Early Cretaceous deposits
5
Fig. 25 A correlation between Chalk Units in the Danish Central Graben, the chronostratigraphic time table, and the formal Chalk Formations established by Deegan \& Scull (1977) for the Northern and Central North Sea area ....

Fig. 26 Evolution of chalk deposition. a) Thicknesses of the six Chalk Units illustrating the development of the Chalk Group in relation to time and location in the Graben area. b) Areal distribution of the chalk deposit zones 1 to 6 deduced from the chalk depositions ................. 50-51

Fig. 27 Distribution and thickness of a) Chalk1 Unit and b) Chalk-2 Unit ........

Fig. 28 Distribution and thickness of a) Chalk3 Unit and b) Chalk-4 Unit ........

Fig. 29 Distribution and thickness of a) Chalk5 Unit and b) Chalk-6 Unit ........

Fig. 30 Lithological units forming top of the Chalk Group ................

Fig. 31 Palinspastic profile of the Late Cretaceous-Danian deposits ........

Fig. 32 Distribution and thickness of the North Sea Marl ..................

Fig. 33 Distribution and thickness of a) the CEN-1 Unit and b) the CEN-2 Unit

Fig. 34 Distribution and thickness of a) the CEN-3 Unit and b) the CEN-4 Unit

Fig. 35 Distribution and thickness of a) the CEN-5 Unit and b) the CEN-6 Unit

Fig. 36 Palinspastic profile of the Cenozoic de-

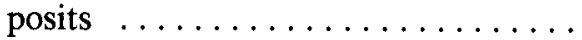

Fig. 37 Temperature profiles calculated from borehole measurements of the Adda-1, E-1, E-4, G-1, H-1, and I-1 wells ...

Fig. 38 Vitrinite reflection trends in the E-1, I$1, \mathrm{M}-8$, and $\mathrm{U}-1$ wells ......... 76

Fig. 39 Calcite/dolomite ratio in the O-1, A-2, G-1, V-1, Q-1, and W-1 wells .....

Fig. 40 Calcite/dolomite ratio in the M-8, E-1, and $\mathrm{I}-1$ wells. . . . . . . . . . 78

Fig. $41 \mathrm{X}$-ray reflection areas in the M-8 and E-1 wells ................. 79

Fig. $42 \mathrm{X}$-ray reflection areas in the I-1 well 80

Fig. 43 Orogano-chemical parameters for the I-1 and M-8 wells ........... 
Fig. 44 Composition of extracts of the I- 1 and M-8 wells ............. 83

Fig. 45 Gas-chromatograms for the M-8 well 84

Fig. 46 Gas-chromatograms for the I-1 well . 85

Fig. 47 Regional subdivision of the Central Graben area based on structural styles

Fig. 48 Schematic diagrams of hydrocarbon traps ..................................

Fig. 49 The reservoir potential of a) Early Carboniferous and $b$ ) Rotliegendes sections in the Central Graben area ........

Fig. 50 The reservoir potential of a) Zechstein and b) the Bacton Group .........

Fig. 51 The reservoir potential of a) the J-2 Unit, Lower Member and b) the J-2 Unit, Upper Member ...........

Fig. 52 The reservoir potential of a) W-1 Unit and $b)$ the J-4 Unit ............

Fig. 53 The reservoir potential of a) the LC-1 Unit and b) the Valhall Formation ..

Fig. 54 Porosity v.s. depth of the Chalk Group in the G-1, M-1, P-1, Q-1, and Ruth-1 88 96 97 wells and a normal compaction curve plotted for comparison on a diagram from Scholle (1977b) . . . . . . . . 101

Fig. 55 Porosity v.s. depth plot of the Chalk Group in selected Central Graben wells $\ldots \ldots \ldots \ldots \ldots \ldots \ldots$.

Fig. 56 Porosity v.s. depth plot of the Chalk 103 Group. Data points are grouped and related to the Chalk deposit zones in Central Graben. ...............

Fig. 57 Schematic presentation of porosity ranges in the Chalk Group related to Chalk deposit zones in the Central Graben. ... . . . . . . . . . . . . . . 104

Fig. 58 Permeability distribution of Chalk

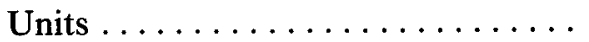

Fig. 59 Core porosity v.s. permeability plot (semi-log) from the E-2 and Ruth-1 wells a) E-2 well. Non fractured chalk with a linear correlation. b) Ruth-1 well. Probably fractured chalk with a non-linear correlation $\ldots \ldots \ldots \ldots . \quad 106$ 